\title{
DESIGN AND CONVERGENCE OF AFEM IN H(DIV)
}

\author{
J. MANUEL CASCON \\ Departamento de Matemáticas, Universidad de Salamanca, \\ 37008 Salamanca, Spain \\ casbar@usal.es \\ RICARDO H. NOCHETTO \\ Department of Mathematics \\ and Institute of Physical Science and Technology, \\ University of Maryland, College Park, MD 20742, USA \\ rhn@math.umd.edu \\ KUNIBERT G. SIEBERT \\ Institut für Mathematik, Universitätsraße 14, \\ 86159 Augsburg, Germany \\ siebert@math.uni-augsburg.de \\ Received 17 August 2006 \\ Revised 13 March 2007 \\ Communicated by F. Brezzi
}

\begin{abstract}
We design an adaptive finite element method (AFEM) for mixed boundary value problems associated with the differential operator $A-\nabla \operatorname{div}$ in $H(\operatorname{div}, \Omega)$. For $A$ being a variable coefficient matrix with possible jump discontinuities, we provide a complete a posteriori error analysis which applies to both Raviart-Thomas $\mathbb{R T}^{n}$ and BrezziDouglas-Marini $\mathbb{B D M}^{n}$ elements of any order $n$ in dimensions $d=2,3$. We prove a strict reduction of the total error between consecutive iterates, namely a contraction property for the sum of energy error and oscillation, the latter being solution-dependent. We present numerical experiments for $\mathbb{R T}^{n}$ with $n=0,1$ and $\mathbb{B D M}^{1}$ which document the performance of AFEM and corroborate as well as extend the theory.
\end{abstract}

Keywords: A posteriori error estimate; error reduction; oscillation; convergence; multigrid preconditioning.

AMS Subject Classification: 65N15, 65N30, 35J25

\section{Introduction}

The space $H(\operatorname{div}, \Omega)$ of square-integrable vector fields with square-integrable divergence is ubiquitous in problems arising in fluid and solid mechanics. ${ }^{5,13}$ It occurs, in particular, in the solution of second order elliptic partial differential equations (PDE) by first order least-squares methods or by mixed methods with augmented Lagrangians. The differential operator $I-\nabla \operatorname{div}$, with appropriate boundary 
conditions, may be considered as a natural realization of the underlying inner product structure of $H(\operatorname{div}, \Omega)$.

We consider more general second order elliptic operators over the Lipschitz polyhedral domain $\Omega$ of $\mathbb{R}^{d}$ with $d=2,3$ and boundary $\partial \Omega:=\Gamma_{\text {ess }} \cup \Gamma_{\text {nat }}$

$$
\mathfrak{L} \mathbf{p}:=A \mathbf{p}-\nabla \operatorname{div} \mathbf{p}=F \quad \text { in } \Omega
$$

with essential boundary condition $\mathbf{p} \cdot \boldsymbol{\nu}=0$ on $\Gamma_{\text {ess }}$. We assume that the matrix $A$ is symmetric, uniformly positive definite, and piecewise Lipschitz with respect to a given coarse triangulation $\mathcal{T}^{0}$ of $\Omega$ into triangles or tetrahedra; hence $A$ may exhibit jump discontinuities aligned with $\mathcal{T}^{0}$. This matrix may account for material properties of porous media, an important area of application of mixed methods. The essential and natural parts of $\partial \Omega, \Gamma_{\text {ess }}$ and $\Gamma_{\text {nat }}$, are supposed to match the partition $\mathcal{T}^{0}$ as well.

To write $(1.1)$ weakly we set $\mathbb{Q}:=H(\operatorname{div}, \Omega)$ and introduce the energy space

$$
\mathbb{Q}_{0}=H_{0, \Gamma_{\text {ess }}}(\operatorname{div}, \Omega)=\left\{\mathbf{q} \in H(\operatorname{div}, \Omega)|\mathbf{q} \cdot \boldsymbol{\nu}|_{\Gamma_{\text {ess }}}=0 \text { on } \Gamma_{\text {ess }}\right\},
$$

along with the (coercive and continuous) bilinear form $a$ : $\mathbb{Q} \times \mathbb{Q} \rightarrow \mathbb{R}$

$$
a(\mathbf{p}, \mathbf{q}):=\langle A \mathbf{p}, \mathbf{q}\rangle_{\Omega}+\langle\operatorname{div} \mathbf{p}, \operatorname{div} \mathbf{q}\rangle_{\Omega} \quad \forall \mathbf{p}, \mathbf{q} \in \mathbb{Q} .
$$

Hereafter, we denote by $\langle\cdot, \cdot\rangle_{\omega}$ the $L^{2}$-scalar product in $\omega$ for any measurable subset $\omega \subset \Omega$, and use boldface for vector-valued functions. If $\mathbb{Q}_{0}^{*}$ denotes the dual space of $\mathbb{Q}_{0}$, we assume that $F \in \mathbb{Q}_{0}^{*}$ is given by

$$
F(\mathbf{q}):=-\left\langle f_{1}, \operatorname{div} \mathbf{q}\right\rangle_{\Omega}+\left\langle\mathbf{f}_{\mathbf{2}}, \mathbf{q}\right\rangle_{\Omega}+\langle g, \mathbf{q} \cdot \boldsymbol{\nu}\rangle_{\Gamma_{\text {nat }}} \quad \forall \mathbf{q} \in \mathbb{Q}_{0}
$$

where

$f_{1}$ is piecewise $H^{1}$ over $\mathcal{T}^{0}$,

$\mathbf{f}_{\mathbf{2}}$ is piecewise $H$ (rot) in $2 \mathrm{~d}$, respectively, $H(\mathbf{c u r l})$ in $3 \mathrm{~d}$ over $\mathcal{T}^{0}$,

and $g \in H^{1}\left(\Gamma_{\text {nat }}\right)$.

The weak formulation of (1.1) reads

$$
\mathbf{p} \in \mathbb{Q}_{0}: a(\mathbf{p}, \mathbf{q})=F(\mathbf{q}) \quad \forall \mathbf{q} \in \mathbb{Q}_{0} .
$$

To understand the smoothing properties of operator $\mathfrak{L}$, it is convenient to use the Helmholtz decomposition $\mathbf{p}=\nabla \phi+\operatorname{curl} \Psi$, which is orthogonal in $L^{2}$. We see that, if $\mathbf{f}_{\mathbf{2}}=$ curl $\mathbf{f}$, then $\phi$ satisfies the elliptic PDE $-\Delta \phi+\phi=f_{1}$ whereas $\Psi=\mathrm{f}$. We thus conclude that $\mathfrak{L}$ has a typical elliptic smoothing in the gradient component of $\mathbb{Q}$ but behaves as the identity (and so without smoothing) in the orthogonal complement.

On the basis of this crucial observation, we provide a complete a posteriori error analysis for (1.1)-(1.3a) in Secs. 5 and 7. To this end, we resort to suitable quasi-Helmholtz decompositions in dimensions $d=2,3$ and thereby get around a convexity requirement on $\Omega$. This analysis is valid for a variable coefficient matrix $A$, and general boundary conditions. This, together with the fact that we enforce 
the least possible refinement compatible with error and oscillation control, leads to a solution-dependent oscillation. Our results apply to both the Raviart-Thomas $\mathbb{R T}^{n}$ and Brezzi-Douglas-Marini $\mathbb{B D M}^{n}$ discretization of any order $n$ in dimensions $d=2,3$. We recall the definition of these spaces in Sec. 2 .

We then formulate an adaptive finite element method (AFEM) in Sec. 3 of the form

$$
\text { SOLVE } \rightarrow \text { ESTIMATE } \rightarrow \text { MARK } \rightarrow \text { REFINE }
$$

and describe these procedures in detail. If $\mathcal{T}^{k}$ is the current mesh, then ESTIMATE computes element indicators of error and oscillation, which provide upper and lower bounds for the energy error. The procedure MARK uses Dörfler marking for error and oscillation, and REFINE iteratively bisects $\mathcal{T}^{k}$ to create the new conforming mesh $\mathcal{T}^{k+1}$ satisfying the so-called Interior Node Property. We then prove convergence of AFEM in Sec. 4. In fact, we show a strict total error reduction between consecutive steps, that is we prove that AFEM is a contraction for the (scaled) sum of energy error and oscillation. The basic ingredients of AFEM for the proof are:

- an a posteriori error estimator with a global upper bound for the error in terms of the error estimator and a discrete local lower bound for the energy between consecutive discrete solutions;

- Dörfler's marking for both energy error and oscillation;

- nested consecutive triangulations, $\mathcal{T}^{k} \subset \mathcal{T}^{k+1}$, with $\mathcal{T}^{k+1}$ having one node in the interior of each marked element of $\mathcal{T}^{k}$ as well as its sides.

As a consequence, we are able to prove a global error reduction estimate up to oscillation and a global oscillation reduction up to error reduction. A tricky aspect of the approach is that oscillation is solution-dependent and so it does not decouple from the energy error. To circumvent this difficulty, we proceed as in Chen-Feng ${ }^{10}$ and Mekchay-Nochetto. ${ }^{17}$ Our approach exhibits an optimal convergence rate, a statement we do not prove in this paper. However, extending the new results of Ref. 8 to this case, we can show an optimal error decay in terms of the degrees of freedom (DOFs). ${ }^{9}$

We also present several numerical experiments in Secs. 6 and 7. They are meant not only to verify the theory, but also to check optimal error decay as well as the multigrid solver performance for highly graded meshes and discontinuous coefficients in terms of the number of DOFs. We show experiments for both $\mathbb{R T}^{n}$ (for $n=0,1)$ and $\mathbb{B D M}^{1}$ elements in dimensions $d=2,3$, and compare their relative merits. We also investigate the role of oscillation.

The paper is organized as follows. In Sec. 2 we discuss further the weak formulation of (1.1) and introduce its discretization. In Sec. 3 we present AFEM and describe the procedures SOLVE, ESTIMATE, MARK, and REFINE. We state without proof some key properties of the estimator and oscillation and use them to prove convergence of AFEM in Sec. 4. In Sec. 5 we construct in $2 \mathrm{~d}$ an efficient and reliable a posteriori error estimator and prove the key properties of error and oscillation 
reduction estimates. We present several numerical examples that illustrate the theory in Sec. 6. Finally, in Sec. 7 we extend the a posteriori analysis to $3 \mathrm{~d}$ and present corresponding numerical experiments.

\section{Properties of the Bilinear Form and Discretization}

Let $d=2,3$ denote the space dimension and let $\Omega \subset \mathbb{R}^{d}$ be a bounded polyhedron. We assume that for $d=2$ the domain $\Omega$ and $\Gamma_{\text {nat }}$ are connected and that for $d=3$ $\Omega$ is topologically equivalent to a ball and $\Gamma_{\text {nat }}=\emptyset$. We introduce the norms

$$
\|\mathbf{q}\|_{\operatorname{div}, \omega}^{2}:=\|\mathbf{q}\|_{\omega}^{2}+\|\operatorname{div} \mathbf{q}\|_{\omega}^{2} \text { and }\|\mathbf{q}\|_{\omega}^{2}:=\left\|A^{\frac{1}{2}} \mathbf{q}\right\|_{\omega}^{2}+\|\operatorname{div} \mathbf{q}\|_{\omega}^{2}
$$

for all $\mathbf{q} \in \mathbb{Q}$, where for $\omega \subset \Omega$ we denote by $\|\cdot\|_{\omega}$ the $L^{2}\left(\omega ; \mathbb{R}^{2}\right)$ norm for vectorvalued and the $L^{2}(\omega)$ norm for scalar-valued functions. The norm $\|\cdot\|_{\operatorname{div}, \Omega}$ is the standard norm on $\mathbb{Q}=H(\operatorname{div}, \Omega)$ and $\|\cdot\|_{\Omega}$ is the energy norm associated with (1.4). Hence, the energy norm is equivalent to the $H(\operatorname{div}, \Omega)$-norm, i.e. there are constants $0<c_{a} \leq C_{a}$ such that

$$
c_{a}\|\mathbf{q}\|_{\operatorname{div}, \Omega}^{2} \leq\|\mathbf{q}\|_{\Omega}^{2} \leq C_{a}\|\mathbf{q}\|_{\operatorname{div}, \Omega}^{2} \quad \text { for all } \mathbf{q} \in \mathbb{Q},
$$

where $c_{a}$ and $C_{a}$ depend on the smallest, respectively the largest eigenvalue of $A$ over $\Omega$. This, in turn, implies that $a: \mathbb{Q}_{0} \times \mathbb{Q}_{0} \rightarrow \mathbb{R}$ is a coercive and continuous bilinear form in $\mathbb{Q}_{0}$, and hence, (1.4) has a unique weak solution $\mathbf{p} \in \mathbb{Q}_{0}$.

Let $\mathcal{T}^{k}$ be a conforming shape-regular triangulation of $\Omega$ and denote by $\mathbb{P}^{n}$ the set of all polynomials of degree $\leq n$. Now, let $\mathbb{Q}^{k} \subset \mathbb{Q}$ be either the Raviart-Thomas elements of order $n \geq 0$ over $\mathcal{T}^{k}$, i.e.

$$
\mathbb{Q}^{k}=\mathbb{R} \mathbb{T}^{n}\left(\mathcal{T}^{k}\right):=\left\{\mathbf{q} \in \mathbb{Q} \mid \mathbf{q}_{\mid T} \in\left(\mathbb{P}^{n}\left(T ; \mathbb{R}^{d}\right)+\boldsymbol{x} \mathbb{P}^{n}(T)\right) \forall T \in \mathcal{T}^{k}\right\},
$$

or the Brezzi-Douglas-Marini elements of order $n \geq 1$, i.e.

$$
\mathbb{Q}^{k}=\mathbb{B D M}^{n}\left(\mathcal{T}^{k}\right):=\left\{\mathbf{q} \in \mathbb{Q} \mid \mathbf{q}_{\mid T} \in \mathbb{P}^{n}\left(T ; \mathbb{R}^{d}\right) \forall T \in \mathcal{T}^{k}\right\} .
$$

The discrete space incorporating the essential boundary condition is finally defined to be $\mathbb{Q}_{0}^{k}=\mathbb{R} \mathbb{T}_{0}^{n}\left(\mathcal{T}^{k}\right)=\mathbb{R} \mathbb{T}^{n}\left(\mathcal{T}^{k}\right) \cap \mathbb{Q}_{0}$ or $\mathbb{Q}_{0}^{k}=\mathbb{B D M} \mathbb{M}_{0}^{n}\left(\mathcal{T}^{k}\right)=\mathbb{B D M} \mathbb{M}^{n}\left(\mathcal{T}^{k}\right) \cap \mathbb{Q}_{0}$.

Then, the discrete formulation of (1.4) reads

$$
\mathbf{P}_{k} \in \mathbb{Q}_{0}^{k}: a\left(\mathbf{P}_{k}, \mathbf{Q}_{k}\right)=F\left(\mathbf{Q}_{k}\right) \quad \forall \mathbf{Q}_{k} \in \mathbb{Q}_{0}^{k} .
$$
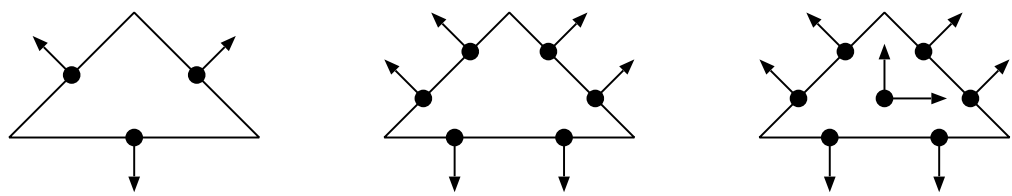

Fig. 1. DOFs for $\mathbb{R T}^{0}, \mathbb{B D M}^{1}$, and $\mathbb{R T}^{1}$ on a triangle $T$. 
Coercivity and continuity of the bilinear form $a$ are inherited from $\mathbb{Q}_{0}$ to any subspace $\mathbb{Q}_{0}^{k}$, implying existence and uniqueness of the discrete solution $\mathbf{P}_{k}$ of (2.1).

\section{Adaptive Algorithm AFEM}

In this section we discuss the basic adaptive loop (1.5) for approximating the solution $\mathbf{p}$ to problem (1.1). Given a grid $\mathcal{T}^{k}$, SOLVE computes the discrete solution $\mathbf{P}_{k}$ to (2.1). The procedure ESTIMATE calculates error and oscillation indicators for all $T \in \mathcal{T}^{k}$ depending on the computed solution and data. The indicators are used by the procedure MARK to make a judicious selection of elements to be refined. Finally, the procedure REFINE divides the selected elements and creates a conforming refinement $\mathcal{T}^{k+1}$ of $\mathcal{T}^{k}$, thereby guaranteeing that $\mathcal{T}^{k}$ and $\mathcal{T}^{k+1}$ are nested. We now present a complete description of these procedures for problem (1.1).

\subsection{Procedure SOLVE: Algebraic solution of the discrete problem}

Given a mesh $\mathcal{T}^{k}$, and the solution $\mathbf{P}_{k-1}$ from the old grid as an initial guess for an iterative solver, the procedure SOLVE is an efficient direct or iterative solver for computing the discrete solution $\mathbf{P}_{k}$ on $\mathcal{T}^{k}$, where we assume that we can solve (2.1) exactly:

$$
\mathbf{P}_{k}:=\operatorname{SOLVE}\left(\mathcal{T}^{k}, \mathbf{P}_{k-1}\right)
$$

\subsection{Procedure ESTIMATE: A Posteriori estimation of error and oscillation}

This procedure computes the error and oscillation indicators, $\eta_{k}(T)$ and $\operatorname{osc}_{k}(T)$, for all $T \in \mathcal{T}^{k}$. Their definitions, given in (5.8) and (5.12) below, are immaterial at this point. The indicators are local a posteriori quantities, i.e. they are computed using only information from the discrete solution and data in a neighborhood of the element $T$. For any subset $\hat{\mathcal{T}}^{k} \subset \mathcal{T}^{k}$ we set

$$
\eta_{k}^{2}\left(\hat{\mathcal{T}}^{k}\right):=\sum_{T \in \hat{\mathcal{T}}^{k}} \eta_{k}^{2}(T), \quad \operatorname{Osc}_{k}^{2}\left(\hat{\mathcal{T}}^{k}\right):=\sum_{T \in \hat{\mathcal{T}}^{k}} \operatorname{osc}_{k}^{2}(T)
$$

and use the convention $\eta_{k}(\hat{\omega})=\eta_{k}\left(\hat{\mathcal{T}}^{k}\right), \operatorname{osc}_{k}(\hat{\omega})=\operatorname{osc}_{k}\left(\hat{\mathcal{T}}^{k}\right)$ if $\hat{\omega}=\cup_{T \in \hat{\mathcal{T}}^{k}} T$, and $\eta_{k}(\emptyset)=\operatorname{osc}_{k}(\emptyset)=0$.

The convergence proof centers around the following two properties of the error estimator. The first one is the global upper bound for the true error $\left\|\mathbf{p}-\mathbf{P}_{k}\right\|_{\Omega}$ by the error estimator $\eta_{k}\left(\mathcal{T}^{k}\right)$ proved in Theorem 5.1:

$$
\left\|\mathbf{p}-\mathbf{P}_{k}\right\|_{\Omega}^{2} \leq C_{1} \eta_{k}^{2}\left(\mathcal{T}^{k}\right),
$$

where the constant $C_{1}$ only depends on the shape-regularity of $\mathcal{T}^{k}, \Omega$, and $1 / c_{a}$.

The second property is a discrete local lower bound for the local error reduction $\left\|\mathbf{P}_{k+1}-\mathbf{P}_{k}\right\|_{\omega_{T}}$ by the indicator $\eta_{k}(T)$, where $\omega_{T} \subset \Omega$ is a patch that consists of 
$T$ and its (at most $d$ ) direct neighbors $T^{\prime} \in \mathcal{T}^{k}$. The lower bound involves the oscillation term $\operatorname{osc}_{k}\left(\omega_{T}\right)$. For obtaining such a bound, an element $T \in \mathcal{T}^{k}$ and its direct neighbors in $\omega_{T}$ need a minimal refinement depth; compare with Refs. 19 and 20:

Interior Node Property: An element $T \in \mathcal{T}^{k}$ fulfills the Interior Node Property with respect to $\mathcal{T}^{k+1}$ if all elements in $\omega_{T}$ as well as all sides of $T$ contain a vertex of $\mathcal{T}^{k+1} \backslash \mathcal{T}^{k}$ in their interior.

The Interior Node Property for an element $T \in \mathcal{T}^{k}$ allows us to prove in Theorem 5.2 and Corollary 5.1 the discrete local lower bound

$$
\bar{C}_{2} \eta_{k}^{2}(T) \leq\left\|\mathbf{P}_{k+1}-\mathbf{P}_{k}\right\|_{\omega_{T}}^{2}+\operatorname{osc}_{k}^{2}\left(\omega_{T}\right),
$$

where $\bar{C}_{2}>0$ only depends on the shape-regularity of $\mathcal{T}^{k+1}$, and $C_{a}$. Due to the structure of the underlying problem (1.1), the oscillation indicators $\operatorname{osc}_{k}(T)$ depend on the discrete solution $\mathbf{P}_{k}$. This complicates the convergence proof in Sec. 4; compare with Refs. 10 and 17.

In summary, the procedure ESTIMATE computes the local indicators $\eta_{k}(T)$ and oscillation terms $\operatorname{Osc}_{k}(T)$ for all elements $T \in \mathcal{T}^{k}$ :

$$
\left\{\eta_{k}(T), \operatorname{osc}_{k}(T)\right\}_{T \in \mathcal{T}^{k}}:=\operatorname{ESTIMATE}\left(\mathcal{T}^{k}, \mathbf{P}_{k}\right)
$$

\subsection{Procedure MARK: Dörfler's marking strategy}

On the basis of the computed values $\left\{\eta_{k}(T), \operatorname{osc}_{k}(T)\right\}_{T \in \mathcal{T}^{k}}$, the procedure MARK generates a set of marked elements subject to refinement. A key ingredient in the convergence proof is an error and oscillation reduction property. Such a property can be derived by selecting sufficiently many elements for refinement. This is quantified by Dörfler's marking strategy, ${ }^{11}$ which we apply to the estimator $\eta_{k}\left(\mathcal{T}^{k}\right)$ as well as to the oscillation $\operatorname{osc}_{k}\left(\mathcal{T}^{k}\right)$ as advocated by Morin et al. ${ }^{19,20}$ :

Marking Strategy: Let $0<\theta_{\mathrm{est}}, \theta_{\mathrm{osc}}<1$ be given parameters.

Mark any subset $\mathcal{T}_{\text {est }}^{k} \subset \mathcal{T}^{k}$ such that $\eta_{k}\left(\mathcal{T}_{\text {est }}^{k}\right) \geq \theta_{\text {est }} \eta_{k}\left(\mathcal{T}^{k}\right)$;

Set $\mathcal{T}_{\mathrm{osc}}^{k}:=\left\{T \in \mathcal{T}^{k} \mid T \subset \omega_{T^{\prime}}\right.$ for some $\left.T^{\prime} \in \mathcal{T}_{\text {est }}^{k}\right\}$

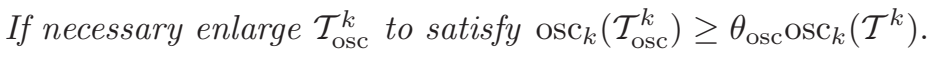

The idea is to select subsets of the triangulation $\mathcal{T}^{k}$ whose element contributions sum up to a fixed amount of the total. In practice, the subsets are chosen as small as possible by collecting the biggest values in order to only introduce a small number of new DOFs in the next mesh. Therefore, the procedure MARK uses the Marking Strategy to select the subsets $\mathcal{T}_{\text {est }}^{k}, \mathcal{T}_{\text {osc }}^{k}$ according to (3.4):

$$
\left\{\mathcal{T}_{\text {est }}^{k}, \mathcal{T}_{\text {osc }}^{k}\right\}:=\operatorname{MARK}\left(\theta_{\text {est }}, \theta_{\text {osc }},\left\{\eta_{k}(T), \operatorname{osc}_{k}(T)\right\}_{T \in \mathcal{T}^{k}}\right)
$$




\subsection{Procedure REFINE: Refinement by bisection}

For the refinement of $\mathcal{T}^{k}$ we rely on iterative or recursive bisection algorithms; compare with Refs. 3, 18 and 21. Starting from a conforming triangulation $\mathcal{T}^{0}$ we construct a sequence of nested, conforming, and uniform shape-regular triangulations $\left\{\mathcal{T}^{k}\right\}_{k \geq 0}$ by local refinement of selected elements. Especially, all constants involving the shape-regularity of $\mathcal{T}^{k}$, like the constants $C_{1}$ in (3.1) and $\bar{C}_{2}$ in (3.3), only depend on $\mathcal{T}^{0}$.

Nesting of the meshes implies nesting of the discrete spaces, i.e. $\mathbb{Q}^{k} \subset \mathbb{Q}^{k+1}$ which in turn entails the orthogonality

$$
\left\|\mathbf{p}-\mathbf{P}_{k+1}\right\|_{\Omega}^{2}=\left\|\mathbf{p}-\mathbf{P}_{k}\right\|_{\Omega}^{2}-\left\|\mathbf{P}_{k+1}-\mathbf{P}_{k}\right\|_{\Omega}^{2}
$$

a crucial property of the energy norm and an essential ingredient of the convergence proof. Equation (3.5) is a direct consequence of the Pythagoras theorem applied to $\mathbf{p}-\mathbf{P}_{k}=\left(\mathbf{p}-\mathbf{P}_{k+1}\right)+\left(\mathbf{P}_{k+1}-\mathbf{P}_{k}\right)$ and the Galerkin orthogonality $a\left(\mathbf{p}-\mathbf{P}_{k+1}, \mathbf{P}_{k+1}-\mathbf{P}_{k}\right)=0 .{ }^{11,19}$ From (3.5) we deduce that the error reduction between two consecutive discrete solutions is exactly $\left\|\mathbf{P}_{k+1}-\mathbf{P}_{k}\right\|_{\Omega}$.

In 2 d, applying at least $2 \frac{1}{2}$ bisections to an element $T \in \mathcal{T}^{k}$ implies the existence of vertices of $\mathcal{T}^{k+1} \backslash \mathcal{T}^{k}$ in the interior of $T$ and all its edges; see Fig. 2. In $3 \mathrm{~d}$, bisecting a tetrahedron $T$ at least six times ensures new vertices in the interior of $T$ as well as in all its faces. For $T \in \mathcal{T}_{\text {est }}^{k}$ we thus refine all elements $T^{\prime} \subset \omega_{T}$ by applying at least $2 \frac{1}{2}(2 \mathrm{~d})$, respectively $6(3 \mathrm{~d})$ bisections. This implies the Interior Node Property for $T \in \mathcal{T}_{\text {est }}^{k}$ and, invoking the discrete local lower bound (3.3), yields the lower bound for the total error reduction

$$
C_{2} \eta_{k}^{2}\left(\mathcal{T}_{\text {est }}^{k}\right) \leq\left\|\mathbf{P}_{k+1}-\mathbf{P}_{k}\right\|_{\Omega}^{2}+\operatorname{osc}_{k}^{2}\left(\mathcal{T}^{k}\right)
$$

with $C_{2}:=\frac{\bar{C}_{2}}{d+1}$ thanks to the finite overlap of the patches $\omega_{T}$ (see Remark 5.3).

Bisecting all elements $T \in \mathcal{T}_{\text {osc }}^{k}$ at least once, implies the oscillation reduction

$$
\operatorname{osc}_{k+1}^{2}\left(\mathcal{T}^{k+1}\right) \leq \rho \operatorname{osc}_{k}^{2}\left(\mathcal{T}^{k}\right)+C_{3}\left\|\mathbf{P}_{k+1}-\mathbf{P}_{k}\right\|_{\Omega}^{2}
$$

proven in Lemma 5.3. The constant $0<\rho<1$ depends on $\theta_{\text {osc }}$ and the minimal number of bisections used for the refinement of $T \in \mathcal{T}_{\text {osc }}^{k}$ and $C_{3}$ depends on the shape-regularity, $C_{a}$, and the element-wise $W_{\infty}^{1}$-norm of $A$.
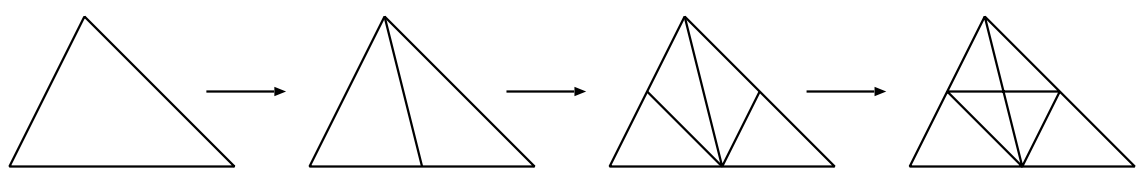

Fig. 2. Refinement of a triangle using $2 \frac{1}{2}$ bisections. After the second bisectioning step, only the two elements at the interior edge are bisected once more. 
In summary, given a mesh $\mathcal{T}^{k}$ and the sets $\mathcal{T}_{\text {est }}^{k}$ and $\mathcal{T}_{\text {osc }}^{k}$ of marked elements, the procedure REFINE creates a conforming refinement $\mathcal{T}^{k+1}$ of $\mathcal{T}^{k}$ ensuring the interior node property for all $T \in \mathcal{T}_{\text {est }}^{k}$ and bisecting all $T \in \mathcal{T}_{\text {osc }}^{k}$ at least once:

$$
\mathcal{T}^{k+1}:=\operatorname{REFINE}\left(\mathcal{T}^{k}, \mathcal{T}_{\text {est }}^{k}, \mathcal{T}_{\text {osc }}^{k}\right)
$$

\subsection{The adaptive algorithm AFEM}

Finally, we summarize the adaptive algorithm (see also Refs. 19, 20 and 17):

AFEM Choose parameters $0<\theta_{\text {est }}, \theta_{\text {osc }}<1$;

(1) Pick up an initial mesh $\mathcal{T}^{0}$, set $k:=0$.

(2) $\mathbf{P}_{k}:=\operatorname{SOLVE}\left(\mathcal{T}^{k}, \mathbf{P}_{k-1}\right)$

(3) $\left\{\eta_{k}(T), \operatorname{osc}_{k}(T)\right\}_{T \in \mathcal{T}^{k}}:=\operatorname{ESTIMATE}\left(\mathcal{T}^{k}, \mathbf{P}_{k}\right)$

(4) $\left\{\mathcal{T}_{\text {est }}^{k}, \mathcal{T}_{\text {osc }}^{k}\right\}:=\operatorname{MARK}\left(\theta_{\text {est }}, \theta_{\text {osc }},\left\{\eta_{k}(T), \operatorname{osc}_{k}(T)\right\}_{T \in \mathcal{T}^{k}}\right)$

(5) $\mathcal{T}^{k+1}:=\operatorname{REFINE}\left(\mathcal{T}^{k}, \mathcal{T}_{\text {est }}^{k}, \mathcal{T}_{\text {osc }}^{k}\right)$

(6) Update $k \leftarrow k+1$, and go to step (2).

\section{Convergence}

In this section we prove convergence of AFEM. Since error and oscillation couple we proceed as Chen-Feng ${ }^{10}$ and Mekchay-Nochetto. ${ }^{17}$ We give a complete proof below for the sake of completeness.

Theorem 4.1. (Convergence of AFEM) Let $\mathbf{p}$ be the true solution to (1.1) and let $\left\{\mathcal{T}^{k}, \mathbf{P}_{k}\right\}_{k \geq 0}$ be the sequence of graded triangulations together with the discrete solutions of (2.1) produced by AFEM.

Then, there exist constants $0<\alpha<1$ and $\beta>0$ such that for two consecutive iterations we get

$$
\left\|\mathbf{p}-\mathbf{P}_{k+1}\right\|_{\Omega}^{2}+\beta \operatorname{osc}_{k+1}^{2}\left(\mathcal{T}^{k+1}\right) \leq \alpha\left(\left\|\mathbf{p}-\mathbf{P}_{k}\right\|_{\Omega}^{2}+\beta \operatorname{osc}_{k}^{2}\left(\mathcal{T}^{k}\right)\right),
$$

whence, if $C_{0}:=\left\|\mathbf{p}-\mathbf{P}_{0}\right\|_{\Omega}^{2}+\beta \operatorname{osc}_{k}^{2}\left(\mathcal{T}^{0}\right)$,

$$
\left\|\mathbf{p}-\mathbf{P}_{k}\right\|_{\Omega}^{2}+\beta \operatorname{osc}_{k+1}^{2}\left(\mathcal{T}^{k+1}\right) \leq C_{0} \alpha^{k} \rightarrow 0, \quad k \rightarrow \infty .
$$

Proof. We use the notation $\mathbf{e}_{k}:=\mathbf{p}-\mathbf{P}_{k}$ and $\mathbf{E}_{k}:=\mathbf{P}_{k+1}-\mathbf{P}_{k}$. We first employ the upper bound (3.1) and the marking (3.4a) for the estimator, which results in the lower bound (3.6) and allow us to write

$$
\left\|\mathbf{e}_{k}\right\|_{\Omega}^{2} \leq C_{1} \eta_{k}^{2}\left(\mathcal{T}^{k}\right) \leq \frac{C_{1}}{\theta_{\text {est }}^{2}} \eta_{k}^{2}\left(\mathcal{T}_{\text {est }}^{k}\right) \leq \frac{C_{1}}{C_{2} \theta_{\text {est }}^{2}}\left(\left\|\mathbf{E}_{k}\right\|_{\Omega}^{2}+\operatorname{osc}_{k}^{2}\left(\mathcal{T}^{k}\right)\right) .
$$


Defining the constant $\Lambda:=\theta_{\text {est }}^{2} C_{2} / C_{1}$ we convert the above expression into

$$
\left\|\mathbf{E}_{k}\right\|_{\Omega}^{2} \geq \Lambda\left\|\mathbf{e}_{k}\right\|_{\Omega}^{2}-\operatorname{osc}_{k}^{2}\left(\mathcal{T}^{k}\right) .
$$

We rewrite $(3.5)$ for a constant $\delta \in(0,1)$ to be chosen later as

$$
\left\|\mathbf{e}_{k+1}\right\|_{\Omega}^{2}=\left\|\mathbf{e}_{k}\right\|_{\Omega}^{2}-\delta\left\|\mathbf{E}_{k}\right\|_{\Omega}^{2}-(1-\delta)\left\|\mathbf{E}_{k}\right\|_{\Omega}^{2} .
$$

Now, replace $\delta\left\|\mathbf{E}_{k}\right\|_{\Omega}^{2}$ via (4.1) to obtain

$$
\left\|\mathbf{e}_{k+1}\right\|_{\Omega}^{2} \leq \alpha_{1}\left\|\mathbf{e}_{k}\right\|_{\Omega}^{2}+\delta \operatorname{osc}_{k}^{2}\left(\mathcal{T}^{k}\right)-(1-\delta)\left\|\mathbf{E}_{k}\right\|_{\Omega}^{2}
$$

with $\alpha_{1}=1-\delta \Lambda<1$. We multiply the oscillation reduction (3.7) by $\beta:=(1-\delta) / C_{3}$ and add it to (4.2) to remove its last term:

$$
\left\|\mathbf{e}_{k+1}\right\|_{\Omega}^{2}+\beta \operatorname{osc}_{k+1}^{2}\left(\mathcal{T}^{k+1}\right) \leq \alpha_{1}\left\|\mathbf{e}_{k}\right\|_{\Omega}^{2}+\left(\delta \beta^{-1}+\rho\right) \beta \operatorname{osc}_{k}^{2}\left(\mathcal{T}^{k}\right) .
$$

For any $\alpha_{2} \in(\rho, 1)$ and $\delta=\left(\alpha_{2}-\rho\right) /\left(C_{3}+\alpha_{2}-\rho\right) \in(0,1)$ we have

$$
\delta \beta^{-1}+\rho=C_{3} \delta(1-\delta)^{-1}+\rho=\alpha_{2}
$$

and thus

$$
\left\|\mathbf{p}-\mathbf{P}_{k+1}\right\|_{\Omega}^{2}+\beta \operatorname{osc}_{k+1}^{2}\left(\mathcal{T}^{k+1}\right) \leq \alpha_{1}\left\|\mathbf{p}-\mathbf{P}_{k}\right\|_{\Omega}^{2}+\alpha_{2} \beta \operatorname{osc}_{k}^{2}\left(\mathcal{T}^{k}\right)
$$

with $\alpha_{1}=1-\theta_{\text {est }}^{2} C_{2}\left(\alpha_{2}-\rho\right) /\left(C_{1}\left(C_{3}+\alpha_{2}-\rho\right)\right)$. The theorem now follows upon taking $\alpha=\max \left\{\alpha_{1}, \alpha_{2}\right\}$.

\section{A Posteriori Error Estimate and Oscillation Reduction}

We now derive (3.1), (3.6), and (3.7) for $d=2$, which are the key estimates to prove Theorem 4.1. Before doing this, we introduce some notations related to the triangulation $\mathcal{T}^{k}$ that is hereafter used for $d=2,3$.

Let $\mathbb{V}^{k} \subset H^{1}(\Omega)$ be the space of piecewise linear finite elements over $\mathcal{T}^{k}$, i.e. piecewise linear functions. The set of sides (edges/faces) of $\mathcal{T}^{k}$ is denoted by $\mathcal{S}^{k}$ and we split $\mathcal{S}^{k}=\mathcal{S}_{\text {int }}^{k} \cup \mathcal{S}_{\text {ess }}^{k} \cup \mathcal{S}_{\text {nat }}^{k}$ into interior sides, sides on $\Gamma_{\text {ess }}$, and sides on $\Gamma_{\text {nat }}$, respectively. The skeleton $\Sigma_{k}$ of $\mathcal{T}^{k}$ is the union of all sides, i.e. $\Sigma_{k}=\bigcup_{\sigma \in \mathcal{S}^{k}} \sigma$.

Associate to a side $\sigma \in \mathcal{S}^{k}$ a unique unit normal vector $\nu$. In $2 \mathrm{~d}, \boldsymbol{\tau}$ denotes the unit tangent vector such that the determinant of the matrix $[\boldsymbol{\nu}, \boldsymbol{\tau}]$ is bigger than zero and $\partial_{\boldsymbol{\tau}} \psi$ is the tangential derivative of a function $\psi \in H^{1}(\sigma)$. If $\sigma \in \mathcal{S}_{\text {int }}^{k}$ is an interior side then $\omega_{\sigma}$ is the union of the two adjacent elements $T_{1}, T_{2} \in \mathcal{T}^{k}$, and $\llbracket v \rrbracket_{\sigma}$ is the jump of the quantity $v$ across $\sigma$, i.e. $\llbracket v \rrbracket_{\sigma}=v_{\mid T_{1}}-v_{\mid T_{2}}$, where the normal associated with $\sigma$ points from $T_{1}$ to $T_{2}$. For a boundary side $\sigma \in \mathcal{S}_{\text {ess }}^{k} \cup \mathcal{S}_{\text {nat }}^{k}$ we denote by $\omega_{\sigma}=T$ the element $T \in \mathcal{T}^{k}$ containing the side $\sigma$.

Finally, we denote by $h_{k} \in L^{\infty}(\Omega)$ the piecewise constant mesh density function defined by $h_{k \mid T}=|T|^{1 / d}$ for all $T \in \mathcal{T}^{k}$. We abuse notation and use the same symbol $h_{k} \in L^{\infty}\left(\Sigma_{k}\right)$ with $h_{k \mid \sigma}=\left|\omega_{\sigma}\right|^{1 / d}$ for $\sigma \in \mathcal{S}_{\text {int }}^{k}$. The symbol $\preccurlyeq$ used later on denotes lower or equal, up to a constant only depending on the shape-regularity of $\mathcal{T}^{0}$. 


\subsection{Approximation in $H($ div $)$}

A basic ingredient for a sharp a posteriori error estimator is an optimal interpolation operator relying on intrinsic regularity only. To avoid a convexity assumption on $\Omega$, we resort to a quasi-Helmholtz decomposition of $\mathbf{q} \in \mathbb{Q}_{0}$. We shall write $\mathbf{q}$ as a sum of a "smooth" vector field and a curl of an $H^{1}$ function with curl $\psi=$ $\left[-\partial_{x_{2}} \psi, \partial_{x_{1}} \psi\right]^{T}$ and $\operatorname{rot} \mathbf{q}=\partial_{x_{2}} q_{1}-\partial_{x_{1}} q_{2}$ being its adjoint. Both parts will be approximated separately.

Lemma 5.1. (Quasi-Helmholtz decomposition) Let $\Omega \subset \mathbb{R}^{2}$ be a bounded and connected Lipschitz domain such that $\Gamma_{\text {nat }}$ is connected.

Then for all $\mathbf{q} \in \mathbb{Q}_{0}$ there exist $\mathbf{\Phi} \in H_{0, \Gamma_{\mathrm{ess}}}^{1}\left(\Omega ; \mathbb{R}^{2}\right)$, i.e. $\boldsymbol{\Phi}$ has zero trace on $\Gamma_{\mathrm{ess}}$, and $\psi \in H^{1}(\Omega)$ with $\psi \equiv c_{i} \in \mathbb{R}$ for all connected components $\Gamma_{\mathrm{ess}}^{i}$ of $\Gamma_{\mathrm{ess}}$ such that

$$
\mathbf{q}=\mathbf{\Phi}+\operatorname{curl} \psi \quad \text { in } \Omega \quad \text { and } \quad\|\nabla \boldsymbol{\Phi}\|_{\Omega},\|\nabla \psi\|_{\Omega} \leq C\|\mathbf{q}\|_{\text {div }, \Omega} .
$$

Proof. Suppose that $\Gamma_{\text {nat }} \neq \emptyset$. Since $\partial \Omega$ is Lipschitz, given $\mathbf{x}_{1} \in \Gamma_{\text {nat }}$ we can find a coordinate system and a rectangle $\mathcal{O}$ aligned with it and centered at $\mathbf{x}_{1}$ such that $\Gamma_{\text {nat }}$ intersects $\mathcal{O}$ in the vertical sides and $\mathcal{O}$ does not intersect $\Gamma_{\text {ess }}$. Consequently $\Omega \cup \mathcal{O}$ is also Lipschitz and connected.

Let $\delta$ be a smooth function with support on $\mathcal{O} \backslash \Omega$ such that

$$
\int_{\mathcal{O} \backslash \Omega} \delta=\int_{\Omega} \operatorname{div} \mathbf{q},
$$

whence $u:=\operatorname{div} \mathbf{q}-\delta$ has mean zero. In view of Ref. 13 (Corollary I.2.4) there exists a vector field $\widehat{\mathbf{\Phi}} \in H_{0}^{1}\left(\Omega \cup \mathcal{O} ; \mathbb{R}^{2}\right)$ such that

$$
\operatorname{div} \widehat{\mathbf{\Phi}}=u \quad \text { and } \quad\|\widehat{\mathbf{\Phi}}\|_{\Omega \cup \mathcal{O}},\|\nabla \widehat{\mathbf{\Phi}}\|_{\Omega \cup \mathcal{O}} \leq C\|\mathbf{q}\|_{\operatorname{div}, \Omega},
$$

where the constant $C$ depends on $\Omega \cup \mathcal{O}$. If $\boldsymbol{\Phi}$ denotes the restriction of $\widehat{\boldsymbol{\Phi}}$ to $\Omega$, then clearly $\boldsymbol{\Phi} \in H_{0, \Gamma_{\text {ess }}}^{1}\left(\Omega ; \mathbb{R}^{2}\right)$ and $\operatorname{div} \boldsymbol{\Phi}=\operatorname{div} \mathbf{q}$ in $\Omega$.

From $(\mathbf{q}-\boldsymbol{\Phi}) \cdot \boldsymbol{\nu}=0$ on $\Gamma_{\text {ess }}, \operatorname{div}(\mathbf{q}-\boldsymbol{\Phi})=0$ in $\Omega$, and $\Gamma_{\text {nat }}$ connected, we deduce $\langle(\mathbf{q}-\mathbf{\Phi}) \cdot \boldsymbol{\nu}, 1\rangle_{\Gamma^{i}}=0$ for any connected component $\Gamma^{i}$ of $\partial \Omega$. This is equivalent to the existence of a stream function $\psi \in H^{1}(\Omega)$, i.e. $\operatorname{curl} \psi=\mathbf{q}-\mathbf{\Phi}$ (Ref. 13, Theorem I.3.1). Since, $\mathbf{q} \in H_{0, \Gamma_{\text {ess }}}(\operatorname{div}, \Omega)$ and $\boldsymbol{\Phi}=0$ on $\Gamma_{\text {ess }}$, we get for the tangential derivative $\partial_{\boldsymbol{\tau}} \psi=\operatorname{curl} \psi \cdot \boldsymbol{\nu}_{\mid \Gamma_{\mathrm{ess}}}=\mathbf{q} \cdot \boldsymbol{\nu}_{\mid \Gamma_{\mathrm{ess}}}-\boldsymbol{\Phi} \cdot \boldsymbol{\nu}_{\mid \Gamma_{\mathrm{ess}}}=0$ on $\Gamma_{\mathrm{ess}}$. This in turn implies $\psi \equiv c_{i}$ on any connected component $\Gamma_{\text {ess }}^{i}$ of $\Gamma_{\text {ess }}$.

The bound for $\boldsymbol{\Phi}$ is a consequence of (5.2), whereas the bound for $\psi$ results from

$$
\|\nabla \psi\|_{\Omega}=\|\operatorname{curl} \psi\|_{\Omega} \leq\|\mathbf{q}\|_{\Omega}+\|\mathbf{\Phi}\|_{\Omega} \leq C\|\mathbf{q}\|_{\text {div }, \Omega} \cdot
$$

The case $\Gamma_{\text {ess }}=\partial \Omega$ is simpler because now div $\mathbf{q}$ has mean zero and the above proof works without any auxiliary function $\delta$.

On the basis of the quasi-Helmholtz decomposition $\mathbf{q}=\boldsymbol{\Phi}+\operatorname{curl} \psi$ of Lemma 5.1, we now construct an approximation $\mathbf{Q}_{k}$ of $\mathbf{q}$ with suitable approximation properties. To this end, let $\Pi_{k}^{n}$ be the $\mathbb{R T}_{0}^{n}$, respectively $\mathbb{B D M}_{0}^{n}$ interpolation operator, i.e.

$$
\Pi_{k}^{n}: \mathbb{Q}_{0} \cap L^{s}\left(\Omega ; \mathbb{R}^{2}\right) \rightarrow \mathbb{Q}_{0}^{k}
$$


for some $s>2$ (Ref. 5 , p. 125). It satisfies for all functions $\boldsymbol{\Phi} \in H^{1}\left(\Omega ; \mathbb{R}^{2}\right)$

$$
\begin{aligned}
\left\langle\left(\boldsymbol{\Phi}-\Pi_{k}^{n} \boldsymbol{\Phi}\right) \cdot \boldsymbol{\nu}, v\right\rangle_{\sigma} & =0 \quad \forall v \in \mathbb{P}^{n}(\sigma) \quad \text { and } \quad \sigma \in \mathcal{S}^{k}, \\
\left\|h_{k}^{-1}\left(\boldsymbol{\Phi}-\Pi_{k}^{n} \boldsymbol{\Phi}\right)\right\|_{L_{2}\left(\Omega ; \mathbb{R}^{2}\right.} & \preccurlyeq\|\nabla \boldsymbol{\Phi}\|_{\Omega}
\end{aligned}
$$

see Ref. 5 (Sec. III.3, p. 127 and Proposition III.3.6). If $\mathcal{I}_{k}: H^{1}(\Omega) \rightarrow \mathbb{V}^{k}$ denotes the Scott-Zhang interpolation operator such that for any connected component $\Gamma_{\text {ess }}^{i}$

$$
\psi \equiv c_{i} \in \mathbb{R} \quad \text { on } \Gamma_{\mathrm{ess}}^{i} \Longrightarrow \mathcal{I}_{k} \psi \equiv c_{i} \quad \text { on } \Gamma_{\mathrm{ess}}^{i} .
$$

We recall the local approximation property for all $\psi \in H^{1}(\Omega)$ :

$$
\left\|h_{k}^{-1}\left(\psi-\mathcal{I}_{k} \psi\right)\right\|_{L^{2}(\Omega)} \preccurlyeq\|\nabla \psi\|_{\Omega},
$$

see (Ref. 22, Theorem 4.1). It is easy to check that $\operatorname{curl} \mathcal{I}_{k} \psi \in \mathbb{Q}^{k}$ holds.

Using the quasi-Helmholtz decomposition for $\mathbf{q} \in \mathbb{Q}_{0}$ we now define an approximation $\mathbf{Q}_{k}$ to $\mathbf{q}$ via the $\Pi_{k}^{n}$ and $\mathcal{I}_{k}$ interpolation operators.

Lemma 5.2. (Approximation in $\mathrm{H}(\operatorname{div})$ ) For $\mathbf{q} \in \mathbb{Q}_{0}$ let $\mathbf{q}=\mathbf{\Phi}+\operatorname{curl} \psi$ be the quasi-Helmholtz decomposition of Lemma 5.1. Let $\boldsymbol{\Phi}_{k}:=\Pi_{k}^{n} \mathbf{\Phi}$ be the $\mathbb{R T}_{0}^{n}$, resp. $\mathbb{B D M}_{0}^{n}$, interpolant of $\boldsymbol{\Phi}$ and $\boldsymbol{\psi}_{k}:=\mathcal{I}_{k} \psi$ be the Scott-Zhang interpolant of $\psi$ and define

$$
\mathrm{Q}_{k}:=\boldsymbol{\Phi}_{k}+\operatorname{curl} \psi_{k} \in \mathbb{Q}_{0}^{k} .
$$

Then, $\psi-\boldsymbol{\psi}_{k}=0$ on $\Gamma_{\mathrm{ess}}$ and for $\delta \boldsymbol{\Phi}:=\mathbf{\Phi}-\boldsymbol{\Phi}_{k}$ and $\delta \psi:=\psi-\boldsymbol{\psi}_{k}$ we have the (local) interpolation estimates

$$
\begin{gathered}
\left\|h_{k}^{-1} \delta \boldsymbol{\Phi}\right\|_{\Omega}+\left\|h_{k}^{-\frac{1}{2}} \delta \boldsymbol{\Phi}\right\|_{\Sigma_{k}} \preccurlyeq\|\nabla \mathbf{\Phi}\|_{\Omega} \preccurlyeq\|\mathbf{q}\|_{\operatorname{div}, \Omega}, \\
\left\|h_{k}^{-1} \delta \psi\right\|_{\Omega}+\left\|h_{k}^{-\frac{1}{2}} \delta \psi\right\|_{\Sigma_{k}} \preccurlyeq\|\nabla \psi\|_{\Omega} \preccurlyeq\|\mathbf{q}\|_{\operatorname{div}, \Omega} .
\end{gathered}
$$

Proof. The approximation properties are a direct consequence of (5.3b) and (5.4), in conjunction with a scaled trace inequality and (5.1). On each connected component $\Gamma_{\text {ess }}^{i} \subset \Gamma_{\text {ess }}$ we have $\psi \equiv c_{i} \in \mathbb{R}$ and thus by construction $\psi-\mathcal{I}_{k} \psi=0$ on $\Gamma_{\text {ess }}$. Furthermore, $\operatorname{curl} \mathcal{I}_{k} \psi \cdot \boldsymbol{\nu}=\partial_{\boldsymbol{\tau}} \mathcal{I}_{k} \psi=0$ on any $\Gamma_{\text {ess }}^{i}$ which yields $\operatorname{curl} \mathcal{I}_{k} \psi \in \mathbb{Q}_{0}^{k}$.

\subsection{Error representation}

We now derive a representation formula of the error $\mathbf{e}_{k}=\mathbf{p}-\mathbf{P}_{k}$. Recall the definition of the right-hand side $F \in \mathbb{Q}_{0}^{*}$ from (1.3a) together with its regularity properties collected in (1.3b). Consider a decomposition $\mathbf{q}=\mathbf{\Phi}+\operatorname{curl} \psi \in \mathbb{Q}_{0}$ with $\Phi \in \mathbb{Q}_{0}$ and $\psi \in H^{1}(\Omega), \psi=0$ on $\Gamma_{\text {ess. }}$. Then the error-residual relation reads

$$
\begin{aligned}
a\left(\mathbf{e}_{k}, \mathbf{q}\right)= & a\left(\mathbf{e}_{k}, \boldsymbol{\Phi}+\operatorname{curl} \psi\right)=F(\boldsymbol{\Phi}+\operatorname{curl} \psi)-a\left(\mathbf{P}_{k}, \boldsymbol{\Phi}+\operatorname{curl} \psi\right) \\
= & \left\langle\mathbf{f}_{2}-A \mathbf{P}_{k}, \boldsymbol{\Phi}+\operatorname{curl} \psi\right\rangle_{\Omega}-\left\langle f_{1}+\operatorname{div} \mathbf{P}_{k}, \operatorname{div} \boldsymbol{\Phi}\right\rangle_{\Omega} \\
& +\langle g,(\boldsymbol{\Phi}+\operatorname{curl} \psi) \cdot \boldsymbol{\nu}\rangle_{\Gamma_{\text {nat }}},
\end{aligned}
$$


thanks to $\operatorname{div} \operatorname{curl} \psi=0$. We split terms related to $\boldsymbol{\Phi}$ and $\operatorname{curl} \psi$ and integrate element-wise by parts, the terms involving $\operatorname{div} \boldsymbol{\Phi}$ and $\operatorname{curl} \psi$. We employ the boundary values $\boldsymbol{\Phi} \cdot \boldsymbol{\nu}_{\mid \Gamma_{\text {ess }}}=\psi_{\mid \Gamma_{\text {ess }}}=0$, to obtain

$$
\begin{aligned}
a\left(\mathbf{e}_{k}, \mathbf{q}\right)= & \sum_{T \in \mathcal{T}^{k}}\left\langle\nabla f_{1}+\mathbf{f}_{2}-A \mathbf{P}_{k}+\nabla \operatorname{div} \mathbf{P}_{k}, \boldsymbol{\Phi}\right\rangle_{T} \\
& -\sum_{\sigma \in \mathcal{S}_{\mathrm{int}}^{k}}\left\langle\llbracket f_{1}+\operatorname{div} \mathbf{P}_{k} \rrbracket, \boldsymbol{\Phi} \cdot \boldsymbol{\nu}\right\rangle_{\sigma}+\sum_{\sigma \in \mathcal{S}_{\mathrm{nat}}^{k}}\left\langle g-\left(f_{1}+\operatorname{div} \mathbf{P}_{k}\right), \boldsymbol{\Phi} \cdot \boldsymbol{\nu}\right\rangle_{\sigma} \\
& +\sum_{T \in \mathcal{T}^{k}}\left\langle\operatorname{rot}\left(\mathbf{f}_{2}-A \mathbf{P}_{k}\right), \psi\right\rangle_{T}+\sum_{\sigma \in \mathcal{S}_{\mathrm{int}}^{k}}\left\langle\llbracket\left(\mathbf{f}_{2}-A \mathbf{P}_{k}\right) \cdot \boldsymbol{\tau} \rrbracket, \psi\right\rangle_{\sigma} \\
& +\sum_{\sigma \in \mathcal{S}_{\mathrm{nat}}^{k}}\left\langle\partial_{\boldsymbol{\tau}} g+\left(\mathbf{f}_{2}-A \mathbf{P}_{k}\right) \cdot \boldsymbol{\tau}, \psi\right\rangle_{\sigma} .
\end{aligned}
$$

Defining elementwise and edgewise residuals to be

$$
\begin{aligned}
& \mathbf{R}_{k \mid T}:=\nabla f_{1}+\mathbf{f}_{2}-A \mathbf{P}_{k}+\nabla \operatorname{div} \mathbf{P}_{k}, \quad T \in \mathcal{T}^{k}, \\
& J_{k \mid \sigma}:= \begin{cases}-\llbracket f_{1}+\operatorname{div} \mathbf{P}_{k} \rrbracket_{\sigma} & \sigma \in \mathcal{S}_{\text {int }}^{k}, \\
0 & \sigma \in \mathcal{S}_{\mathrm{ess}}^{k}, \\
g-\left(f_{1}+\operatorname{div} \mathbf{P}_{k}\right) & \sigma \in \mathcal{S}_{\text {nat }}^{k},\end{cases} \\
& r_{k \mid T}:=\operatorname{rot}\left(\mathbf{f}_{2}-A \mathbf{P}_{k}\right) \quad T \in \mathcal{T}^{k}, \\
& j_{k \mid \sigma}:= \begin{cases}\llbracket\left(\mathbf{f}_{2}-A \mathbf{P}_{k}\right) \cdot \boldsymbol{\tau} \rrbracket_{\sigma} & \sigma \in \mathcal{S}_{\mathrm{int}}^{k}, \\
0 & \sigma \in \mathcal{S}_{\mathrm{ess}}^{k}, \\
\partial_{\tau} g+\left(\mathbf{f}_{2}-A \mathbf{P}_{k}\right) \cdot \boldsymbol{\tau} & \sigma \in \mathcal{S}_{\text {nat }}^{k} .\end{cases}
\end{aligned}
$$

We deduce the error representation

$$
a\left(\mathbf{p}-\mathbf{P}_{k}, \mathbf{q}\right)=\left\langle\mathbf{R}_{k}, \boldsymbol{\Phi}\right\rangle_{\Omega}+\left\langle J_{k}, \boldsymbol{\Phi} \cdot \boldsymbol{\nu}\right\rangle_{\Sigma_{k}}+\left\langle r_{k}, \psi\right\rangle_{\Omega}+\left\langle j_{k}, \psi\right\rangle_{\Sigma_{k}},
$$

where $\mathbf{q}=\boldsymbol{\Phi}+\operatorname{curl} \psi \in \mathbb{Q}_{0}$ is decomposed into $\boldsymbol{\Phi} \in \mathbb{Q}_{0}$ and $\psi \in H^{1}(\Omega)$ with $\psi_{\mid \Gamma_{\text {ess }}}=0$. Moreover, if $\mathbf{q} \in \mathbb{Q}_{0}^{k+1}$, then (5.6) yields

$$
a\left(\mathbf{P}_{k+1}-\mathbf{P}_{k}, \mathbf{q}\right)=\left\langle\mathbf{R}_{k}, \boldsymbol{\Phi}\right\rangle_{\Omega}+\left\langle J_{k}, \boldsymbol{\Phi} \cdot \boldsymbol{\nu}\right\rangle_{\Sigma_{k}}+\left\langle r_{k}, \psi\right\rangle_{\Omega}+\left\langle j_{k}, \psi\right\rangle_{\Sigma_{k}},
$$

thanks to Galerkin orthogonality $a\left(\mathbf{p}-\mathbf{P}_{k+1}, \mathbf{q}\right)=0$. We note that (5.6) is the starting point for proving the upper bound, whereas (5.7) leads to the discrete lower bound. This is achieved in the next two sections.

\subsection{Upper bound}

We now use the error representation (5.6) in conjunction with Galerkin orthogonality and the approximation properties (5.5), to derive the upper bound.

Theorem 5.1. (Upper bound) Let $\mathbf{p} \in \mathbb{Q}_{0}$ be the solution of (1.4) and let $\mathbf{P}_{k} \in \mathbb{Q}_{0}^{k}$ be the discrete solution of (2.1). For $T \in \mathcal{T}^{k}$ define the local error indicators

$$
\eta_{k}^{2}(T):=\left\|h_{k} \mathbf{R}_{k}\right\|_{T}^{2}+\left\|h_{k}^{\frac{1}{2}}\left(J_{k}-\bar{J}_{k}\right)\right\|_{\partial T}^{2}+\left\|h_{k} r_{k}\right\|_{T}^{2}+\left\|h_{k}^{\frac{1}{2}} j_{k}\right\|_{\partial T}^{2},
$$

where $\bar{J}_{k \mid \sigma}$ is the $L_{2}(\sigma)$ projection of $J_{k \mid \sigma}$ onto $\mathbb{P}^{n}(\sigma)$ for $\sigma \in \mathcal{S}^{k}$. 
Then, there exist a constant $C_{1}>0$, only depending on the shape-regularity of $\mathcal{T}^{0}, \Omega$, and $1 / c_{a}$, such that the following a posteriori error estimate for the true error holds

$$
\left\|\mathbf{p}-\mathbf{P}_{k}\right\|_{\Omega}^{2} \leq C_{1} \eta_{k}^{2}\left(\mathcal{T}^{k}\right)=C_{1} \sum_{T \in \mathcal{T}^{k}} \eta_{k}^{2}(T) .
$$

Proof. Define $\mathbf{e}_{k}:=\mathbf{p}-\mathbf{P}_{k}$ and let $\mathbf{e}_{k}=\boldsymbol{\Phi}+\operatorname{curl} \psi$ be its quasi-Helmholtz decomposition by Lemma 5.1 and let $\mathbf{Q}_{k}=\mathbf{\Phi}_{k}+\operatorname{curl} \psi_{k}$ be its approximation by Lemma 5.2. Now, set $\mathbf{q}:=\mathbf{e}_{k}-\mathbf{Q}_{k}=\delta \boldsymbol{\Phi}+\operatorname{curl} \delta \psi:=\left(\boldsymbol{\Phi}-\boldsymbol{\Phi}_{k}\right)+\operatorname{curl}\left(\psi-\boldsymbol{\psi}_{k}\right) \in$ $\mathbb{Q}_{0}$, where $\delta \boldsymbol{\Phi} \in \mathbb{Q}_{0}$ and $\delta \psi \in H^{1}(\Omega)$ with $\delta \psi=0$ on $\Gamma_{\text {ess }}$.

By Galerkin orthogonality $a\left(\mathbf{e}_{k}, \mathbf{e}_{k}\right)=a\left(\mathbf{e}_{k}, \mathbf{e}_{k}-\mathbf{Q}_{k}\right)=a\left(\mathbf{e}_{k}, \mathbf{q}\right)$ and thus (5.6) implies

$$
\left\|\mathbf{e}_{k}\right\|_{\Omega}^{2}=a\left(\mathbf{e}_{k}, \mathbf{q}\right)=\left\langle\mathbf{R}_{k}, \delta \boldsymbol{\Phi}\right\rangle_{\Omega}+\left\langle J_{k}, \delta \boldsymbol{\Phi} \cdot \boldsymbol{\nu}\right\rangle_{\Sigma_{k}}+\left\langle r_{k}, \delta \psi\right\rangle_{\Omega}+\left\langle j_{k}, \delta \psi\right\rangle_{\Sigma_{k}} .
$$

Property (5.3a) of the $\mathbb{R T}_{0}^{n}$, respectively $\mathbb{B D M}_{0}^{n}$ interpolant $\boldsymbol{\Phi}_{k}$ of $\boldsymbol{\Phi}$ and definition of $\bar{J}_{k}$ yield $\left\langle J_{k}, \delta \boldsymbol{\Phi} \cdot \boldsymbol{\nu}\right\rangle_{\Sigma_{k}}=\left\langle J_{k}-\bar{J}_{k}, \delta \boldsymbol{\Phi} \cdot \boldsymbol{\nu}\right\rangle_{\Sigma_{k}}$. Hence,

$$
\left\|\mathbf{e}_{k}\right\|_{\Omega}^{2}=\left\langle\mathbf{R}_{k}, \delta \boldsymbol{\Phi}\right\rangle_{\Omega}+\left\langle J_{k}-\bar{J}_{k}, \delta \boldsymbol{\Phi} \cdot \boldsymbol{\nu}\right\rangle_{\Sigma_{k}}+\left\langle r_{k}, \delta \psi\right\rangle_{\Omega}+\left\langle j_{k}, \delta \psi\right\rangle_{\Sigma_{k}} .
$$

Invoking Cauchy-Schwarz inequality we infer that

$$
\begin{aligned}
\left\|\mathbf{e}_{k}\right\|_{\Omega}^{2} \leq & \left\|h_{k} \mathbf{R}_{k}\right\|_{\Omega}\left\|h_{k}^{-1} \delta \boldsymbol{\Phi}\right\|_{\Omega}+\left\|h_{k}^{\frac{1}{2}}\left(J_{k}-\bar{J}_{k}\right)\right\|_{\Sigma_{k}}\left\|h_{k}^{-\frac{1}{2}} \delta \boldsymbol{\Phi}\right\|_{\Sigma_{k}} \\
& +\left\|h_{k} r_{k}\right\|_{\Omega}\left\|h_{k}^{-1} \delta \psi\right\|_{\Omega}+\left\|h_{k}^{\frac{1}{2}} j_{k}\right\|_{\Sigma_{k}}\left\|h_{k}^{-\frac{1}{2}} \delta \psi\right\|_{\Sigma_{k}} .
\end{aligned}
$$

All terms involving $\delta \boldsymbol{\Phi}$ and $\delta \psi$ can be estimated by $\left\|\mathbf{e}_{k}\right\|_{\operatorname{div}, \Omega}$, using estimates (5.5a) and (5.5b) of Lemma 5.2. The asserted upper bound (5.9) then directly follows from $\left\|\mathbf{e}_{k}\right\|_{\operatorname{div}, \Omega} \leq c_{a}^{-1 / 2}\left\|\mathbf{e}_{k}\right\|_{\Omega}$ and definition of $\eta_{k}\left(\mathcal{T}^{k}\right)$.

Remark 5.1. (Jump residuals) Let $\sigma \in \mathcal{S}_{\text {int }}^{k}$ be a side such that $\sigma \not \subset \sigma_{0}$ for any $\sigma_{0} \in \mathcal{S}_{\text {int }}^{0}$. Consequently, we get $\llbracket f_{1} \rrbracket_{\sigma}=0$ whence $J_{k}-\bar{J}_{k}=0$ on $\sigma$. Similarly, for such $\sigma$ we have $j_{k}=-\llbracket A \mathbf{P}_{k} \rrbracket_{\sigma}$. Especially, for $f_{1} \in H^{1}(\Omega)$ or $\mathbf{f}_{2} \in H(\operatorname{rot} ; \Omega)$ we have $J_{k}-\bar{J}_{k}=0$, respectively, $j_{k}=-\llbracket A \mathbf{P}_{k} \rrbracket_{\sigma}$ for all $\sigma \in \mathcal{S}_{\text {int }}^{k}$.

\subsection{Local lower bound}

We now establish a (discrete) lower bound for the error $\mathbf{P}_{k+1}-\mathbf{P}_{k}$ upon taking advantage of properties of discrete bubble functions. These functions are piecewise polynomials on the refinement $\mathcal{T}^{k+1}$ of $\mathcal{T}^{k}$. We start with a crucial geometric result.

Lemma 5.3. (Discrete Bubble Function) Let $T$ be a triangle that is bisected $2 \frac{1}{2}$ times as depicted in Fig. 3. For $n=0,1$ let $\mathbb{B}^{n}(T)$ be the Raviart-Thomas space of order $n$ over the refinement of $T$ with zero normal trace on $\partial T$.

Then for all polynomials $\mathbf{q} \in \mathbb{P}^{n}\left(T ; \mathbb{R}^{2}\right)$ of degree $n$ there exists a bubble function $\mathbf{b} \in \mathbb{B}^{n}(T)$ such that

$$
\|\mathbf{q}\|_{T}^{2} \preccurlyeq\langle\mathbf{q}, \mathbf{b}\rangle_{T} \quad \text { and } \quad\|\mathbf{b}\|_{T} \preccurlyeq\|\mathbf{q}\|_{T},
$$

where the constants hidden in $\preccurlyeq$ only depend on the aspect ratio of $T$. 


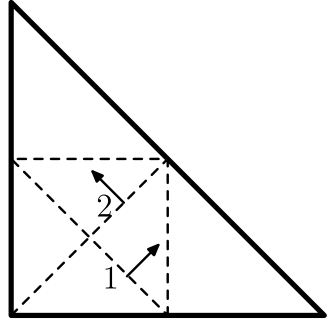

(a)

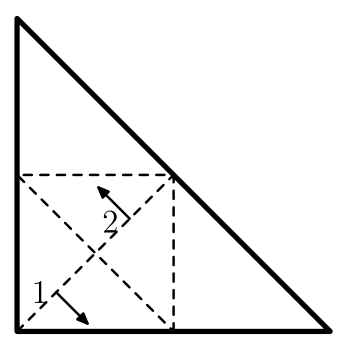

(b)

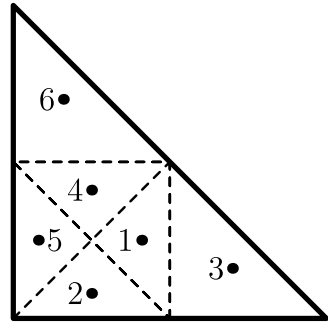

(c)

Fig. 3. Raviart-Thomas basis functions for $2 \frac{1}{2}$ bisections of the reference element $\hat{T}$. (a), (b): nodes of $\mathbb{R T}^{0}$ basis functions $\left\{\boldsymbol{\Phi}_{i}\right\}_{i=1}^{2}$ associated with boundary DOFs $\int_{\sigma_{i}} \boldsymbol{\Phi}_{i}^{T} \boldsymbol{\nu}_{i}=1$ for the given normals $\boldsymbol{\nu}_{i}$ to edges $\sigma_{i}$. (c): nodes of $\mathbb{R T}^{1}$ basis functions $\left\{\boldsymbol{\Phi}_{i}\right\}_{i=1}^{6}$ associated with the interior DOFs $\int_{T_{i}} \boldsymbol{\Phi}_{i}^{T} \mathbf{e}_{1}=1$ in each sub-triangle $T_{i}$ of $\hat{T}$. Both (a) and (c) are used in Lemma 5.3, whereas (b) is a counterexample (see Remark 5.2).

\section{Proof.}

1. For the reference triangle $\hat{T}$ depicted in Fig. 3, we show below that for all $\hat{\mathbf{q}} \in$ $\mathbb{P}^{n}\left(\hat{T} ; \mathbb{R}^{2}\right)$ there exists a $\hat{\mathbf{b}} \in \mathbb{B}^{n}(\hat{T})$ with

$$
\|\hat{\mathbf{q}}\|_{\hat{T}}^{2}=\langle\hat{\mathbf{q}}, \hat{\mathbf{b}}\rangle_{\hat{T}}, \quad \text { and } \quad\|\hat{\mathbf{b}}\|_{\hat{T}} \preccurlyeq\|\hat{\mathbf{q}}\|_{\hat{T}} .
$$

Let $F: \hat{T} \rightarrow T$ be invertible and affine and denote by $\mathfrak{A}:=|D F|^{-1 / 2} D F$ a scaled Jacobian of $F$. Then

$$
\lambda_{\min }\|\hat{\mathbf{q}}\|_{\hat{T}}^{2}=\lambda_{\min } \int_{\hat{T}} \hat{\mathbf{q}}^{T} \hat{\mathbf{q}} \leq \int_{\hat{T}} \hat{\mathbf{q}}^{T} \mathfrak{A}^{-1} \mathfrak{A}^{-T} \hat{\mathbf{q}}=\left\|\mathfrak{A}^{-T} \hat{\mathbf{q}}\right\|_{\hat{T}}^{2} \leq \lambda_{\max }\|\hat{\mathbf{q}}\|_{\hat{T}}^{2}
$$

for all $\hat{\mathbf{q}} \in \mathbb{P}^{n}\left(\hat{T} ; \mathbb{R}^{2}\right)$, since the eigenvalues $\lambda_{\min }, \lambda_{\max }$ of the positive definite matrix $\mathfrak{A}^{-1} \mathfrak{A}^{-T}$ are of order 1 and only depend on the aspect ratio of $T$.

For $\mathbf{q} \in \mathbb{P}^{n}\left(T ; \mathbb{R}^{2}\right)$ let $\hat{\mathbf{b}} \in \mathbb{B}^{n}(\hat{T})$ be the bubble function of (5.11) associated to $\hat{\mathbf{q}}:=\mathfrak{A}^{T} \mathbf{q} \circ F \in \mathbb{P}^{n}\left(\hat{T} ; \mathbb{R}^{2}\right)$. Then holds

$$
\frac{1}{|D F|}\|\mathbf{q}\|_{T}^{2}=\left\|\mathfrak{A}^{-T} \hat{\mathbf{q}}\right\|_{\hat{T}}^{2} \preccurlyeq\|\hat{\mathbf{q}}\|_{\hat{T}}^{2}=\langle\hat{\mathbf{q}}, \hat{\mathbf{b}}\rangle_{\hat{T}}=\left\langle\mathfrak{A}^{-T} \hat{\mathbf{q}}, \mathfrak{A} \hat{\mathbf{b}}\right\rangle_{\hat{T}}=\frac{1}{|D F|}\langle\mathbf{q}, \mathbf{b}\rangle_{T}
$$

for $\mathbf{b}:=\mathfrak{A} \hat{\mathbf{b}} \circ F^{-1}$ being a scaled Piola transformation of $\hat{\mathbf{b}}$. The Piola transformation preserves degrees of freedom of Raviart-Thomas elements and hence $\hat{\mathbf{b}} \in$ $\mathbb{B}^{n}(\hat{T})$ implies $\mathbf{b} \in \mathbb{B}^{n}(T)$; see Ref. 5 (Sec. III.1, p. 98). On the other hand,

$$
\frac{1}{|D F|}\|\mathbf{b}\|_{T}^{2}=\|\mathfrak{A} \hat{\mathbf{b}}\|_{\hat{T}}^{2} \preccurlyeq\|\hat{\mathbf{q}}\|_{\hat{T}}^{2} \preccurlyeq\left\|\mathfrak{A}^{-T} \hat{\mathbf{q}}\right\|_{\hat{T}}^{2}=\frac{1}{|D F|}\|\mathbf{q}\|_{T}^{2},
$$

which implies (5.10). It remains to show (5.11) which we do next.

2. In order to simplify notation set $T:=\hat{T}$. Let $\left\{\boldsymbol{\xi}_{i}\right\}_{i=1}^{I}$ be a basis of $\mathbb{P}^{n}\left(T ; \mathbb{R}^{2}\right)$, define the mass matrix $\mathfrak{M}=\left[\left\langle\boldsymbol{\xi}_{i}, \boldsymbol{\xi}_{j}\right\rangle_{T}\right]_{i, j=1}^{I}$, and write

$$
\mathbf{q}=\sum_{i=1}^{I} q_{i} \boldsymbol{\xi}_{i} \quad \text { and set } \quad \mathbf{Q}:=\left[q_{1}, \ldots, q_{I}\right]^{T} .
$$


Below we construct $\left\{\boldsymbol{\Phi}_{i}\right\}_{i=1}^{I} \in \mathbb{B}^{n}(T)$ such that the matrix $\mathfrak{B}=\left[\left\langle\boldsymbol{\xi}_{i}, \boldsymbol{\Phi}_{j}\right\rangle_{T}\right]_{i, j=1}^{I}$ is invertible. If we define

$$
\mathbf{b}=\sum_{i=1}^{I} b_{i} \boldsymbol{\Phi}_{i} \quad \text { with } \mathbf{B}=\left[b_{1}, \ldots, b_{I}\right]^{T}:=\mathfrak{B}^{-1} \mathfrak{M} \mathbf{Q},
$$

then $\mathbf{b} \in \mathbb{B}^{n}(T)$ satisfies (5.11) since $\|\mathbf{q}\|_{T}^{2}=\mathbf{Q}^{T} \mathfrak{M} \mathbf{Q}=\mathbf{Q}^{T} \mathfrak{B B}=\langle\mathbf{q}, \mathbf{b}\rangle_{T}$ and invertibility of $\mathfrak{B}$ together with equivalence of norms on $\mathbb{P}^{n}\left(T ; \mathbb{R}^{2}\right)$ and $\mathbb{B}^{n}(T)$ implies $\|\mathbf{b}\|_{T} \preccurlyeq\|\mathbf{q}\|_{T}$. Next, we prove that it is possible to construct an invertible $\mathfrak{B}$.

3. For $n=0$, let $\boldsymbol{\Phi}_{i}$ be the Raviart-Thomas basis function associated with the interior edge $\sigma_{i}$, for $i=1,2$, with the orientation plotted in Fig. 3(a). For $\boldsymbol{\xi}_{1}=\mathbf{e}_{1}=[1,0]^{T}$ and $\boldsymbol{\xi}_{2}=\mathbf{e}_{2}=[0,1]^{T}, \mathfrak{B}$ is explicitly given as

$$
\mathfrak{B}=\frac{1}{6}\left[\begin{array}{rr}
1 & 1 \\
-1 & 1
\end{array}\right] \text {. }
$$

For $n=1$, let $\left\{\boldsymbol{\Phi}_{i}\right\}_{i=1}^{6}$ be the Raviart-Thomas basis functions associated with the interior DOFs in the sub-elements $T_{i}, i=1, \ldots, 6$, as depicted in Fig. 3(c). If $\left\{\boldsymbol{\xi}_{i}\right\}_{i=1}^{6}$ is the canonical basis of $\mathbb{P}^{1}\left(T ; \mathbb{R}^{2}\right)$, namely $\mathbf{e}_{1}, \mathbf{e}_{2}, x \mathbf{e}_{1}, x \mathbf{e}_{2}, y \mathbf{e}_{1}, y \mathbf{e}_{2}$, then $\mathfrak{B}$ is given by

$$
\mathfrak{B}=\frac{1}{120}\left[\begin{array}{rrrrrr}
0 & 60 & 0 & 26 & 1 & 15 \\
60 & 0 & 15 & -1 & 4 & 0 \\
-60 & 60 & -42 & 40 & -1 & 12 \\
-60 & 60 & -12 & 1 & -40 & 42 \\
0 & -60 & 0 & -4 & 1 & -15 \\
-60 & 0 & -15 & -1 & 26 & 0
\end{array}\right] .
$$

We have $|\mathfrak{B}|=\frac{1}{18}$ for $n=0$, respectively, $|\mathfrak{B}|=\frac{1}{2,400}$ for $n=1$, and thus step 2 implies the assertion.

We are now ready to define for $T \in \mathcal{T}^{k}$ and $\sigma \in \mathcal{S}^{k}$ several oscillation terms, where we use the same symbol $\pi_{k}^{n}$ in different ways, depending on $n$ and its argument. For $\mathbb{R T}^{n}$ we denote by $\overline{\mathbf{R}}_{k \mid T}:=\pi_{k}^{n} \mathbf{R}_{k \mid T}$ the $L^{2}$-projection onto either $\mathbb{P}^{n}\left(T ; \mathbb{R}^{2}\right)$ for $n=0,1$ or onto $\mathbb{R T}^{n-1}(T)$ for $n \geq 2$. For $\mathbb{B D M}^{n}$, we denote by $\pi_{k}^{n} \mathbf{R}_{k \mid T}$ the $L^{2}$-projection onto $\mathbb{P}^{0}\left(T ; \mathbb{R}^{2}\right)$ for $n=1$ or onto $\mathbb{B D M}^{n-1}(T)$ for $n \geq 2$. For all $n \geq 0$, let $\bar{r}_{k \mid T}:=\pi_{k}^{n} r_{k \mid T}$ be the $L^{2}$-projection onto $\mathbb{P}^{n}(T)$ and let $\bar{\jmath}_{k \mid \sigma}:=\pi_{k}^{n} j_{k \mid \sigma}$ be the $L^{2}$-projection onto $\mathbb{P}^{n}(\sigma)$. The oscillation indicator for $T \in \mathcal{T}^{k}$ is now defined to be

$$
\begin{aligned}
\operatorname{osc}_{k}^{2}(T)= & \left\|h_{k}\left(\mathbf{R}_{k}-\overline{\mathbf{R}}_{k}\right)\right\|_{T}^{2}+\left\|h_{k}^{\frac{1}{2}}\left(J_{k}-\bar{J}_{k}\right)\right\|_{\partial T}^{2} \\
& +\left\|h_{k}\left(r_{k}-\bar{r}_{k}\right)\right\|_{T}^{2}+\left\|h_{k}^{\frac{1}{2}}\left(j_{k}-\bar{\jmath}_{k}\right)\right\|_{\partial T}^{2}
\end{aligned}
$$

We first prove a discrete lower bound for the Raviart-Thomas discretization. 
Theorem 5.2. (Discrete local lower bound for $\mathbb{R T}^{n}$ ) For $n \geq 0$ consider the Raviart-Thomas discretization $\mathbb{Q}^{k}=\mathbb{R}^{n}\left(\mathcal{T}^{k}\right)$ and let $\mathbf{P}_{k+1} \in \mathbb{Q}_{0}^{k+1}$ and $\mathbf{P}_{k} \in \mathbb{Q}_{0}^{k}$ be two consecutive solutions of (2.1).

Then, there exists a constant $\bar{C}_{2}>0$, only depending on the shape-regularity of $\mathcal{T}^{0}, \Omega$, and $C_{a}$, such that for all $T \in \mathcal{T}^{k}$ satisfying the Interior Node Property (3.2) we have

$$
\bar{C}_{2} \eta_{k}^{2}(T) \leq\left\|\mathbf{P}_{k+1}-\mathbf{P}_{k}\right\|_{\omega_{T}}^{2}+\operatorname{osc}_{k}^{2}\left(\omega_{T}\right)
$$

where $\omega_{T}$ is the union of the elements in $\mathcal{T}^{k}$ sharing an edge with $T$.

Proof. We split the proof into three steps to account for the residuals $\overline{\mathbf{R}}_{k}, \bar{r}_{k}, \bar{\jmath}_{k}$. Let $\varphi_{T}$ and $\varphi_{\sigma}$ be the piecewise linear basis functions over $\mathcal{T}^{k+1}$ associated with the interior nodes of $T$, respectively $\sigma \subset \partial T$, and set $\mathbf{E}_{k}:=\mathbf{P}_{k+1}-\mathbf{P}_{k}$.

1. Interior residual $\overline{\mathbf{R}}_{k}$. Let $\mathbf{b} \in \mathbb{B}^{n}(T)$ be the bubble function for $\mathbf{q}=\overline{\mathbf{R}}_{k}$ according to Lemma 5.3 if $n=0,1$, or $\mathbf{b}=\varphi_{T} \overline{\mathbf{R}}_{k}$ for $n \geq 2$. After extending $\mathbf{b}$ by zero outside $T$ we see that $\mathbf{b} \in \mathbb{Q}_{0}^{k+1}$, since $T$ was bisected at least $2 \frac{1}{2}$ times. Invoking (5.10) for $n=0,1$ or the fact that $\varphi_{T}>0$ in $T$ is a piecewise polynomial we obtain

$$
\left\|\overline{\mathbf{R}}_{k}\right\|_{T}^{2} \preccurlyeq\left\langle\overline{\mathbf{R}}_{k}, \mathbf{b}\right\rangle_{T}=\left\langle\overline{\mathbf{R}}_{k}-\mathbf{R}_{k}, \mathbf{b}\right\rangle_{T}+\left\langle\overline{\mathbf{R}}_{k}, \mathbf{b}\right\rangle_{T}=\left\langle\overline{\mathbf{R}}_{k}-\mathbf{R}_{k}, \mathbf{b}\right\rangle_{T}+a\left(\mathbf{E}_{k}, \mathbf{b}\right),
$$

where in the last step we have used (5.7) with $\mathbf{q}=\mathbf{\Phi}=\mathbf{b}$. Combining the CauchySchwarz inequality with an inverse estimate, we have

$$
\left\|\overline{\mathbf{R}}_{k}\right\|_{T}^{2} \preccurlyeq\left(\left\|\overline{\mathbf{R}}_{k}-\mathbf{R}_{k}\right\|_{T}+h_{T}^{-1}\left\|\mathbf{E}_{k}\right\|_{T}\right)\|\mathbf{b}\|_{T}
$$

with $h_{T}=h_{k \mid T}$. Since $\|\mathbf{b}\|_{T} \preccurlyeq\left\|\overline{\mathbf{R}}_{k}\right\|_{T}$, either as a consequence of (5.10) for $n=0,1$ or by direct computation for $n \geq 2$, we deduce

$$
\left\|h_{k} \overline{\mathbf{R}}_{k}\right\|_{T}^{2} \preccurlyeq\left\|\mathbf{E}_{k}\right\|_{T}^{2}+\left\|h_{k}\left(\overline{\mathbf{R}}_{k}-\mathbf{R}_{k}\right)\right\|_{T}^{2} .
$$

The same estimate holds true for all adjacent elements in $\omega_{T}$.

2. Interior residual $\bar{r}_{k}$. The function $\psi:=\bar{r}_{k} \varphi_{T} \in H_{0}^{1}(T)$ is a piecewise polynomial of degree $n+1$ on the refinement of $T$, hence $\operatorname{curl} \psi \in \mathbb{Q}_{0}^{k+1}$. Using $\mathbf{q}=\operatorname{curl} \psi$ in (5.7) and employing standard scaling arguments together with $0 \leq \varphi_{T} \leq 1$ we get

$$
\begin{aligned}
\left\|\bar{r}_{k}\right\|_{T}^{2} & \preccurlyeq\left\langle\bar{r}_{k}, \psi\right\rangle_{T}=\left\langle\bar{r}_{k}-r_{k}, \psi\right\rangle_{T}+\left\langle r_{k}, \psi\right\rangle_{T}=\left\langle\bar{r}_{k}-r_{k}, \psi\right\rangle_{T}+a\left(\mathbf{E}_{k}, \operatorname{curl} \psi\right) \\
& \preccurlyeq\left(\left\|\bar{r}_{k}-r_{k}\right\|_{T}+h_{T}^{-1}\left\|\mathbf{E}_{k}\right\|_{T}^{2}\right)\left\|\bar{r}_{k}\right\|_{T} .
\end{aligned}
$$

We thus deduce for $T$ as well as the adjacent elements in $\omega_{T}$

$$
\left\|h_{k} \bar{r}_{k}\right\|_{T}^{2} \preccurlyeq\left\|\mathbf{E}_{k}\right\|_{T}^{2}+\left\|h_{k}\left(\bar{r}_{k}-r_{k}\right)\right\|_{T}^{2} .
$$

3. Jump residual $\bar{\jmath}_{k}$. Let $\sigma \in \mathcal{S}^{k}$ be a side of $T$, and extend $\bar{\jmath}_{k}$ constant along the normal to $\sigma$ so that $\bar{\jmath}_{k} \in \mathbb{P}^{n}\left(\omega_{\sigma}\right)$, where we recall that $\omega_{\sigma}$ is the union of the elements 
containing $\sigma$. We set $\psi:=\bar{\jmath}_{k} \varphi_{\sigma} \in H_{0}^{1}\left(\omega_{\sigma}\right)$ and observe that $\mathbf{q}=\operatorname{curl} \psi \in \mathbb{Q}_{0}^{k+1}$ is an admissible test function in (5.7). Arguing as before, we arrive at

$$
\begin{aligned}
\left\|\bar{\jmath}_{k}\right\|_{\sigma}^{2} & \preccurlyeq\left\langle\bar{\jmath}_{k}, \psi\right\rangle_{\sigma}=\left\langle\bar{\jmath}_{k}-j_{k}, \psi\right\rangle_{\sigma}+a\left(\mathbf{E}_{k}, \operatorname{curl} \psi\right)-\left\langle r_{k}, \psi\right\rangle_{\omega_{\sigma}} \\
& \preccurlyeq\left\|\bar{\jmath}_{k}-j_{k}\right\|_{\sigma}\left\|\bar{\jmath}_{k}\right\|_{\sigma}+\left(h_{T}^{-1}\left\|\mathbf{E}_{k}\right\|_{\omega_{\sigma}}+\left\|r_{k}\right\|_{\omega_{\sigma}}\right)\left\|\bar{\jmath}_{k}\right\|_{\omega_{\sigma}} .
\end{aligned}
$$

Finally, the inverse estimate $\left\|\bar{\jmath}_{k}\right\|_{\omega_{\sigma}} \preccurlyeq h_{T}^{\frac{1}{2}}\left\|\bar{\jmath}_{k}\right\|_{\sigma}$, together with (5.14), yields

$$
\left\|h_{k}^{\frac{1}{2}} \bar{\jmath}_{k}\right\|_{\partial T} \preccurlyeq\left\|\mathbf{E}_{k}\right\|_{\omega_{T}}+\left\|h_{k}\left(\bar{r}_{k}-r_{k}\right)\right\|_{\omega_{T}}+\left\|h_{k}^{\frac{1}{2}}\left(\bar{\jmath}_{k}-j_{k}\right)\right\|_{\partial T} .
$$

This completes the proof.

Remark 5.2. (Order of oscillation) The $L_{2}$ projection of $\mathbf{R}_{k \mid T}$ onto $\mathbb{R T}^{n-1}(T)$ is of order $n-1$, whereas the $L_{2}$ projection onto $\mathbb{P}^{n}\left(T ; \mathbb{R}^{2}\right)$ is of order $n$. Hence, the oscillation term $\left\|h_{k}\left(\mathbf{R}_{k}-\overline{\mathbf{R}}_{k}\right)\right\|_{\Omega}$ is generically of higher order than the estimator for $n \leq 1$ but not for $n \geq 2$. Convergence of AFEM is still granted for all $n \in \mathbb{N}_{0}$.

Using a projection onto $P^{n}\left(T ; \mathbb{R}^{2}\right)$ for all $n$ would need a generalization of the crucial Lemma 5.3 to any $n \in \mathbb{N}_{0}$. The proof hinges on showing that the matrix $\mathfrak{B}$ is invertible. A counting argument yields $N=\operatorname{dim} \mathbb{P}^{n}\left(T ; \mathbb{R}^{2}\right) \leq \operatorname{dim} \mathbb{B}^{n}(T)$ which suggests that one always can choose functions $\left\{\boldsymbol{\Phi}_{i}\right\}_{i=1, \ldots, N} \subset \mathbb{B}^{n}(\hat{T})$ for constructing an invertible matrix $\mathfrak{B}$. However, this simple counting argument does not imply the existence of such $\mathbf{b}$. Indeed, even for $n=0$ choosing $\boldsymbol{\Phi}_{1}, \boldsymbol{\Phi}_{2} \in \mathbb{B}^{0}(\hat{T})$ as depicted in Fig. 3 (b) leads to $|\mathfrak{B}|=0$. This explains our explicit construction of $\mathbf{b} \in \mathbb{B}^{n}(T)$ for $\overline{\mathbf{R}}_{k} \in \mathbb{P}^{n}\left(T ; \mathbb{R}^{2}\right)$ if $n=0,1$ and the use of the general approach $\mathbf{b}=\varphi_{T} \overline{\mathbf{R}}_{k} \in \mathbb{B}^{n}(T)$ for $\overline{\mathbf{R}}_{k} \in \mathbb{R T}^{n-1}(T)$ if $n \geq 2$. This construction is even more useful in $3 \mathrm{~d}$; compare with Sec. 7.4.

The other oscillation terms for $\mathbb{R T}^{n}$ as well as all oscillation terms for $\mathbb{B D M}^{n}$ are generically of higher order.

Corollary 5.1. (Discrete local lower bound for $\mathbb{B D M}^{n}$ ) For $n \geq 0$ consider the Brezzi-Douglas-Marini discretization $\mathbb{Q}^{k}=\mathbb{B D M}^{n}\left(\mathcal{T}^{k}\right)$ and let $\mathbf{P}_{k+1} \in \mathbb{Q}_{0}^{k+1}$ and $\mathbf{P}_{k} \in \mathbb{Q}_{0}^{k}$ be two consecutive solutions of $(2.1)$.

Then there exists a constant $\bar{C}_{2}>0$, only depending on the shape-regularity of $\mathcal{T}^{0}, \Omega$ and $C_{a}$, such that for all $T \in \mathcal{T}^{k}$ satisfying the Interior Node Property (3.2) we have

$$
\bar{C}_{2} \eta_{k}^{2}(T) \leq\left\|\mathbf{P}_{k+1}-\mathbf{P}_{k}\right\|_{\omega_{T}}^{2}+\operatorname{osc}_{k}^{2}\left(\omega_{T}\right)
$$

Proof. For $n=1$ we observe $\mathbb{R T}^{0}(T) \subset \mathbb{B D M}^{1}(T)$ and thus for constant $\overline{\mathbf{R}}_{k}$ the explicit construction of the discrete bubble $\mathbf{b} \in \mathbb{R T}^{0}(T) \subset \mathbb{B D M}^{1}(T)$ in Lemma 5.3 is valid in this case, too. For $n \geq 2$ we use the general construction as for $\mathbb{R}^{n}$, i.e. $\mathbf{b}=\varphi_{T} \overline{\mathbf{R}}_{k} \in \mathbb{B D M}^{n}(T)$. The other terms are estimated as above.

Corollary 5.2. (Local lower bound) Let $\mathbf{p} \in \mathbb{Q}_{0}$ be the solution of (1.4) and let $\mathbf{P}_{k} \in \mathbb{Q}_{0}^{k}$ be the discrete solution of $(2.1)$. 
If $\bar{C}_{2}$ is the constant from Theorem 5.2, respectively Corollary 5.1, then we have

$$
\bar{C}_{2} \eta_{k}^{2}(T) \leq\left\|\mathbf{p}-\mathbf{P}_{k}\right\|_{\omega_{T}}^{2}+\operatorname{osc}_{k}^{2}\left(\omega_{T}\right) \quad \forall T \in \mathcal{T}^{k} .
$$

Proof. It suffices to observe that for all $T \in \mathcal{T}^{k}$ the test functions used in the proof of Theorem 5.2 and Corollary 5.1 are admissible test functions in (5.6). The claim follows by using (5.6) instead of (5.7) for all $T \in \mathcal{T}^{k}$.

Remark 5.3. (Discrete global lower bound) The refinement procedure REFINE assures the Interior Node Property (3.2) for all elements $T \in \mathcal{T}_{\text {est }}^{k}$. Adding (5.13), respectively (5.15), over all elements of $\mathcal{T}_{\text {est }}^{k}$, using the finite overlap of the patches $\omega_{T}$, and setting $C_{2}:=\bar{C}_{2} / 4$ we end up with

$$
C_{2} \eta_{k}^{2}\left(\mathcal{T}_{\text {est }}^{k}\right) \leq\left\|\mathbf{P}_{k+1}-\mathbf{P}_{k}\right\|_{\Omega}^{2}+\operatorname{osc}_{k}^{2}\left(\mathcal{T}^{k}\right) \leq\left\|\mathbf{p}-\mathbf{P}_{k}\right\|_{\Omega}^{2}+\operatorname{osc}_{k}^{2}\left(\mathcal{T}^{k}\right)
$$

where we have used $\left\|\mathbf{P}_{k+1}-\mathbf{P}_{k}\right\|_{\Omega} \leq\left\|\mathbf{p}-\mathbf{P}_{k}\right\|_{\Omega}$ by (3.5).

\subsection{Oscillation reduction}

For $T \in \mathcal{T}^{k}$ the oscillation indicator $\operatorname{osc}_{k}(T)$ is defined via local projection operators $\pi_{k}$ that are not invariant in the space $\mathbb{R}^{n}(T)$, respectively $\mathbb{B D M}^{n}(T)$. This, together with the fact that the matrix $A$ is variable within $T$, and may exhibit discontinuities across $\partial T$, leads to a solution-dependent oscillation. The same situation occurs in Ref. 17 and, therefore, we will follow a similar approach in the study of oscillation reduction. The crucial quantity in our analysis is the local mesh-size reduction factor

$$
\lambda_{T}:=\max \left\{\left\|h_{k+1} h_{k}^{-1}\right\|_{L_{\infty}(T)},\left\|h_{k+1}^{\frac{1}{2}} h_{k}^{-\frac{1}{2}}\right\|_{L_{\infty}(\partial T)}\right\} \leq 1, \quad \forall T \in \mathcal{T}^{k} .
$$

As a consequence, $\lambda_{T}<1$ for all $T \in \mathcal{T}^{k}$ that are bisected at least once and $\lambda_{T} \leq \lambda<1$ for all $T \in \mathcal{T}_{\text {osc }}^{k}$, where $\lambda$ depends on the minimal number of bisections applied to $T \in \mathcal{T}_{\text {osc }}^{k}$.

Lemma 5.4. (Local oscillation bound) There exists a constant $\bar{C}_{3}<\infty$ solely depending on the shape-regularity of $\mathcal{T}^{0}, C_{a}$ and the element-wise $W_{\infty}^{1}$-norm of $A$, such that for any $\delta>0$ and all $T \in \mathcal{T}^{k}$ holds

$$
\operatorname{osc}_{k+1}^{2}(T) \leq(1+\delta) \lambda_{T}^{2} \operatorname{osc}_{k}^{2}(T)+\left(1+\delta^{-1}\right) \bar{C}_{3}\left\|\mathbf{P}_{k+1}-\mathbf{P}_{k}\right\|_{\omega_{T}}^{2} .
$$

Proof. We let $\mathbf{E}_{k}:=\mathbf{P}_{k+1}-\mathbf{P}_{k}$ and denote by $\mathcal{R}_{k}$ any of the residuals $\mathbf{R}_{k}, J_{k}, r_{k}$ or $j_{k}$. Given $T \in \mathcal{T}^{k}$, let $\mathcal{R}_{k+1}$ be defined over a set $K^{\prime} \subset T$ that is either an element $T^{\prime} \in \mathcal{T}^{k+1}$ or a side $\sigma^{\prime} \in \mathcal{S}^{k+1}$. We can write on $K^{\prime}$

$$
\mathcal{R}_{k+1}-\pi_{k+1}^{n} \mathcal{R}_{k+1}=\mathcal{R}_{k}-\pi_{k+1}^{n} \mathcal{R}_{k}+\left(I-\pi_{k+1}^{n}\right)\left(\mathcal{R}_{k+1}-\mathcal{R}_{k}\right) .
$$

We examine the two terms on the right-hand side separately, but first note that the norm of $I-\pi_{k+1}^{n}$ as a linear operator in $L^{2}\left(K^{\prime}\right)$ is bounded by 1 , whence

$$
\left\|\mathcal{R}_{k+1}-\pi_{k+1}^{n} \mathcal{R}_{k+1}\right\|_{K^{\prime}}^{2} \leq(1+\delta)\left\|\mathcal{R}_{k}-\pi_{k+1}^{n} \mathcal{R}_{k}\right\|_{K^{\prime}}^{2}+\left(1+\delta^{-1}\right)\left\|\mathcal{R}_{k+1}-\mathcal{R}_{k}\right\|_{K^{\prime}}^{2}
$$


where $\delta>0$ is entering by Young's inequality. To deal with the first term, we recall that $\pi_{k+1}^{n} \mathcal{R}_{k}$ provides the best $L^{2}$-approximation of $\mathcal{R}_{k}$ over the set $K^{\prime}$. Therefore, given $K \in \mathcal{T}^{k}$ (or $\mathcal{S}^{k}$ ) and a partition of $K$ into elements $K^{\prime} \in \mathcal{T}^{k+1}$ (or $\mathcal{S}^{k+1}$ ), and using definition (5.16) of $\lambda_{K}$ we deduce with $\gamma=1$ (or $\frac{1}{2}$ )

$\left\|h_{k+1}^{\gamma}\left(\mathcal{R}_{k}-\pi_{k+1}^{n} \mathcal{R}_{k}\right)\right\|_{K}^{2} \leq \lambda_{K}^{2} \sum_{K^{\prime} \subset K}\left\|h_{k}^{\gamma}\left(\mathcal{R}_{k}-\pi_{k}^{n} \mathcal{R}_{k}\right)\right\|_{K^{\prime}}^{2}=\lambda_{K}^{2}\left\|h_{k}^{\gamma}\left(\mathcal{R}_{k}-\pi_{k}^{n} \mathcal{R}_{k}\right)\right\|_{K}^{2}$.

For the second term above, we show that $\mathcal{R}_{k+1}-\mathcal{R}_{k}$ is proportional to $\mathbf{E}_{k}$ and its derivatives. We study each residual separately, starting with $\mathbf{R}_{k}$. We have

$$
\mathbf{R}_{k+1}-\mathbf{R}_{k}=-A \mathbf{E}_{k}+\nabla \operatorname{div} \mathbf{E}_{k}
$$

whence, utilizing an inverse estimate on $T^{\prime} \in \mathcal{T}^{k+1}$, we end up with

$$
\left\|h_{k+1} A \mathbf{E}_{k}\right\|_{T^{\prime}}+\left\|h_{k+1} \nabla \operatorname{div} \mathbf{E}_{k}\right\|_{T^{\prime}} \preccurlyeq\left\|A \mathbf{E}_{k}\right\|_{T^{\prime}}+\left\|\operatorname{div} \mathbf{E}_{k}\right\|_{T^{\prime}} \preccurlyeq\left\|\mathbf{E}_{k}\right\|_{T^{\prime}} .
$$

A similar argument applies to $r_{k+1}-r_{k}=-\operatorname{rot}\left(A \mathbf{E}_{k}\right)=-\operatorname{rot} A \cdot \mathbf{E}_{k}-A: \operatorname{curl} \mathbf{E}_{k}$

$$
\left\|h_{k+1}\left(r_{k+1}-r_{k}\right)\right\|_{T^{\prime}} \preccurlyeq\left\|\mathbf{E}_{k}\right\|_{T^{\prime}} .
$$

To analyze the jump residuals, we take $\sigma^{\prime} \in \mathcal{S}^{k+1}$ and let $\omega_{\sigma^{\prime}}$ be its patch in $\mathcal{T}^{k+1}$. Setting $J_{k}=j_{k}=0$ on $\Sigma_{k+1} \backslash \Sigma_{k}$, an inverse inequality finally yields

$$
\left\|h_{k+1}^{\frac{1}{2}}\left(J_{k+1}-J_{k}\right)\right\|_{\sigma^{\prime}}=\left\|h_{k+1}^{\frac{1}{2}} \llbracket \operatorname{div} \mathbf{E}_{k} \rrbracket\right\|_{\sigma^{\prime}} \preccurlyeq\left\|\operatorname{div} \mathbf{E}_{k}\right\|_{\omega_{\sigma^{\prime}}} \preccurlyeq\left\|\mathbf{E}_{k}\right\|_{\omega_{\sigma^{\prime}}},
$$

as well as

$$
\left\|h_{k+1}^{\frac{1}{2}}\left(j_{k+1}-j_{k}\right)\right\|_{\sigma^{\prime}}=\left\|h_{k+1}^{\frac{1}{2}} \llbracket A \mathbf{E}_{k} \cdot \boldsymbol{\tau} \rrbracket\right\|_{\sigma^{\prime}} \preccurlyeq\left\|\mathbf{E}_{k}\right\|_{\omega_{\sigma^{\prime}}} \preccurlyeq\left\|\mathbf{E}_{k}\right\|_{\omega_{\sigma^{\prime}}} .
$$

This proves (5.17) regardless of whether $T \in \mathcal{T}^{k}$ has been refined or not.

Corollary 5.3. (Oscillation reduction) Let $\mathcal{T}^{k+1}$ be a refinement of $\mathcal{T}^{k}$ where all elements in $\mathcal{T}_{\text {osc }}^{k}$ are bisected at least once. Let $\mathbf{P}_{k} \in \mathbb{Q}_{0}^{k}$ and $\mathbf{P}_{k+1} \in \mathbb{Q}_{0}^{k+1}$ be the discrete solutions of (2.1). Then there exists a constant $\rho<1$, depending on $\theta_{\mathrm{osc}}$ and the mesh-size reduction factor $\lambda<1$, as well as a constant $C_{3}$, depending on the shape-regularity of $\mathcal{T}^{0}, C_{a}$ and the element-wise $W_{\infty}^{1}$-norm of $A$, such that

$$
\operatorname{osc}_{k+1}^{2}\left(\mathcal{T}^{k+1}\right) \leq \rho \operatorname{osc}_{k}^{2}\left(\mathcal{T}^{k}\right)+C_{3}\left\|\mathbf{P}_{k+1}-\mathbf{P}_{k}\right\|_{\Omega}^{2} .
$$

Proof. We observe that $\lambda_{T} \leq \lambda<1$ for all $T \in \mathcal{T}_{\text {osc }}^{k}$ and $\lambda_{T} \leq 1$ for all $T \in \mathcal{T}^{k}$. Hence, for $\mathbf{E}_{k}:=\mathbf{P}_{k+1}-\mathbf{P}_{k}$ and any $\delta>0$ we obtain by Lemma 5.4

$$
\operatorname{osc}_{k+1}^{2}\left(\mathcal{T}^{k+1}\right) \leq(1+\delta) \sum_{T \in \mathcal{T}^{k}} \lambda_{T}^{2} \operatorname{osc}_{k}^{2}(T)+\left(1+\delta^{-1}\right) \bar{C}_{3} \sum_{T \in \mathcal{T}^{k}}\left\|\mathbf{E}_{k}\right\|_{\omega_{T}}^{2} .
$$

By virtue of Dörfler's marking for oscillation (3.4b), we arrive at

$$
\begin{aligned}
\sum_{T \in \mathcal{T}^{k}} \lambda_{T}^{2} \operatorname{Osc}_{k}^{2}(T) & \leq \lambda^{2} \operatorname{osc}_{k}^{2}\left(\mathcal{T}_{\text {osc }}^{k}\right)+\operatorname{osc}_{k}^{2}\left(\mathcal{T}^{k} \backslash \mathcal{T}_{\text {osc }}^{k}\right) \\
& =\left(\lambda^{2}-1\right) \operatorname{osc}_{k}^{2}\left(\mathcal{T}_{\text {osc }}^{k}\right)+\operatorname{osc}_{k}^{2}\left(\mathcal{T}^{k}\right) \leq\left(1-\theta_{\text {osc }}^{2}\left(1-\lambda^{2}\right)\right) \operatorname{osc}_{k}^{2}\left(\mathcal{T}^{k}\right) .
\end{aligned}
$$

Since $1-\theta_{\text {osc }}^{2}\left(1-\lambda^{2}\right)<1$ we now can choose $\delta$ sufficiently small such that

$$
\rho:=(1+\delta)\left(1-\theta_{\text {osc }}^{2}\left(1-\lambda^{2}\right)\right)<1 \text {. }
$$


The assertion follows upon realizing that $\sum_{T \in \mathcal{T}^{k}}\left\|\mathbf{E}_{k}\right\|_{\omega_{T}}^{2} \leq 4\left\|\mathbf{E}_{k}\right\|_{\Omega}^{2}$, because of the finite overlap of patches $\omega_{T}$, and finally taking $C_{3}:=\left(1+\delta^{-1}\right) 4 \bar{C}_{3}$.

\section{Numerical Experiments}

In this section we present some numerical examples which corroborate the convergence of AFEM, quantify the role of oscillation, and exhibit an optimal convergence rate; the latter will be proved in Ref. 9. The experiments have been performed with the finite element toolbox ALBERTA using refinement by recursive bisection. ${ }^{21} \mathrm{We}$ have implemented $\mathbb{R T}^{0}, \mathbb{R T}^{1}$, and $\mathbb{B D M}{ }^{1}$. The finite element space on an element $T$ is realized by a function space on a reference element $\hat{T}$, and the Piola transformation from $\hat{T}$ to $T$. Furthermore, the multigrid solver described in Sec. 6.1 for $\mathbb{R} \mathbb{T}^{0}$, the estimator $\eta_{k}\left(\mathcal{T}^{k}\right)$ and oscillation term $\operatorname{osc}_{k}\left(\mathcal{T}^{k}\right)$ defined in Sec. 5.2 are implemented. Procedure MARK uses $\theta_{\text {est }}=\theta_{\text {osc }}=0.4$, and procedure REFINE sub-divides all elements $T \in \mathcal{T}_{\text {est }}^{k}$ as well as all direct neighbors $T^{\prime}$ of $T \in \mathcal{T}_{\text {est }}^{k}$ by creating all grandchildren of 3rd generation. This implies the interior node property (3.2) for all $T \in \mathcal{T}_{\text {est }}^{k}$.

\subsection{Multigrid preconditioning}

The efficient solution of the linear algebraic system arising from (2.1) is not straightforward. This difficulty is usual in mixed finite element methods. The development and theoretical justification of fast algorithms for solving such problems have been studied recently. ${ }^{2,14}$ In our case, to solve efficiently the system associated to the operator $\mathfrak{L}:=A-\nabla$ div, we use a $C G$ preconditioning with a $\mathrm{V}$-cycle multigrid developed by Arnold et al. Although theoretically only justified for quasi-uniform triangulations, this method seems also optimal for highly graded meshes, i.e. the number of iterations remains bounded as the number of DOFs grows as long as the coefficient matrix $A$ is quasi-monotone, see Ref. 12. The situation for a checkerboard pattern is much less clear and we refer to Sec. 6.3.

The V-cycle multigrid is defined using an additive Schwarz smoother based on solutions to local problems on finite element stars, i.e. the union of all elements meeting at one mesh vertex. For graded meshes, we introduce the following definition of multigrid levels $\mathcal{M}^{j}$, associated with the nested sequence of meshes $\left\{\mathcal{T}^{k}\right\}_{k \geq 0}$ constructed by the refinement process: each element $T \in \mathcal{T}^{k}$ is assigned a level $\ell(T)$, so that

$$
\ell(T):=0 \quad \text { if } T \in \mathcal{T}^{0} \quad \text { and } \quad \ell(T):=\ell\left(T^{\prime}\right)+1 \quad \text { if } T \text { is child of } T^{\prime} .
$$

The multigrid levels are set to be $\mathcal{M}^{0}:=\mathcal{T}^{0}$ and

$$
\mathcal{M}^{j}:=\{T: \ell(T)=j\} \cup\{T: \ell(T)<j \text { and } T \text { has no children }\} .
$$

Note that $\mathcal{M}^{j}$ is a triangulation of $\Omega$ for all $j$ and that, in general, the multigrid level $\mathcal{M}^{j}$ is different from $\mathcal{T}^{j}$. The smoother of the $\mathrm{V}$-cycle multigrid in the level 
$j$ is defined as a scaled sum of solutions to the restriction of the discrete problem (2.1) to the star at each vertex of $\mathcal{M}^{j}$. On the boundary of a star we use essential boundary condition $\mathbf{p} \cdot \boldsymbol{\nu}=0$, except where the star's boundary coincides with the domain boundary and then the boundary condition of (2.1) is applied.

\subsection{Example 1: L-shaped domain}

Let $\Omega=\{|x|+|y|<1\} \cap\{x<0$ or $y>0\}$ be an L-shaped domain, and $\mathbf{p}:=A^{-1} u$ the solution of (1.1) where

$$
\begin{aligned}
u(r, \theta) & =r^{\frac{2}{3}} \sin \left(\frac{2 \theta}{3}\right)-\frac{r^{2}}{4}, \\
A & =I, \quad f_{1}=1+u, \quad \mathbf{f}_{2}=\mathbf{0}, \quad \mathbf{g}=\mathbf{u}, \quad \partial \Omega=\Gamma_{\text {nat }},
\end{aligned}
$$

and $(r, \theta)$ denote polar coordinates. Note that $u$ is the solution of the elliptic equation $-\Delta u=1$ in $\Omega$.

We solve the problem for both $\mathbb{R T}^{0}$ and $\mathbb{R T}^{1}$. The optimal error decay in terms of the degrees of freedom (DOFs) can be appreciated in Fig. 4. In both cases, oscillation is a higher order term and plays a minor role: The number of elements marked by oscillation is insignificant (always less than 10).

We solve the linear system arising from (2.1) using $\mathbb{R T}^{0}$ by means of PCG with the multigrid preconditioner of Sec. 6.1. We emphasize that in this case the number of iterations of PCG remains bounded as the number of DOFs grows [see Fig. 6(a)], and thus indicates that the theory in Refs. 2 and 14 extends to graded meshes.

\subsection{Example 2: Discontinuous coefficients}

We deal with Kellogg's exact solution of the elliptic problem ${ }^{15}$

$$
-\operatorname{div} A^{-1} \nabla u=0 \quad \text { in } \Omega, \quad u=g \quad \text { on } \partial \Omega,
$$

with $A$ piecewise constant in sectors with a vertex at the origin. In particular, we consider the checkerboard example presented by Morin et al. in Ref. 19, where Kellogg's formulas are written for $\Omega=(-1,1)^{2}$, and $A=a_{1}^{-1} I$ in the first and the third quadrants, whereas $A=a_{2}^{-1} I$ in the second and the fourth quadrants. An exact solution of (6.1) is given in polar coordinates by $u(r, \theta)=r^{\gamma} \mu(\theta)$, where $\mu(\theta)$ is a smooth function depending of $\theta$. We choose $\mathbf{p}:=A^{-1} u$ to be the solution of (1.1) with the following parameters and data

$$
\begin{aligned}
& \gamma=0.25, \quad a_{1} \cong 25.27414236908818, \quad a_{2}=1, \\
& f_{1}=u, \quad \mathbf{f}_{2}=\mathbf{0}, \quad g=u, \quad \Gamma_{\text {nat }}=\partial \Omega,
\end{aligned}
$$

and employ $\mathbb{R T}^{0}$. The checkerboard pattern is the most demanding configuration in terms of regularity and multigrid performance. Multigrid seems to be sensitive to the jumps of $A$ because the number of PCG iterations for $\mathbb{R T}^{0}$ increases slightly 


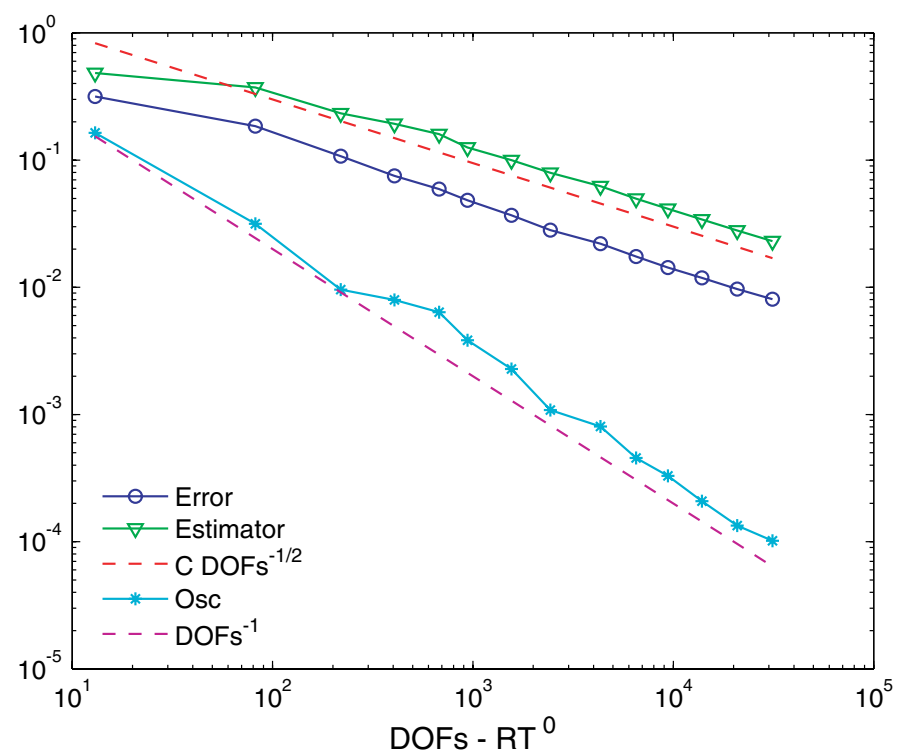

(a)

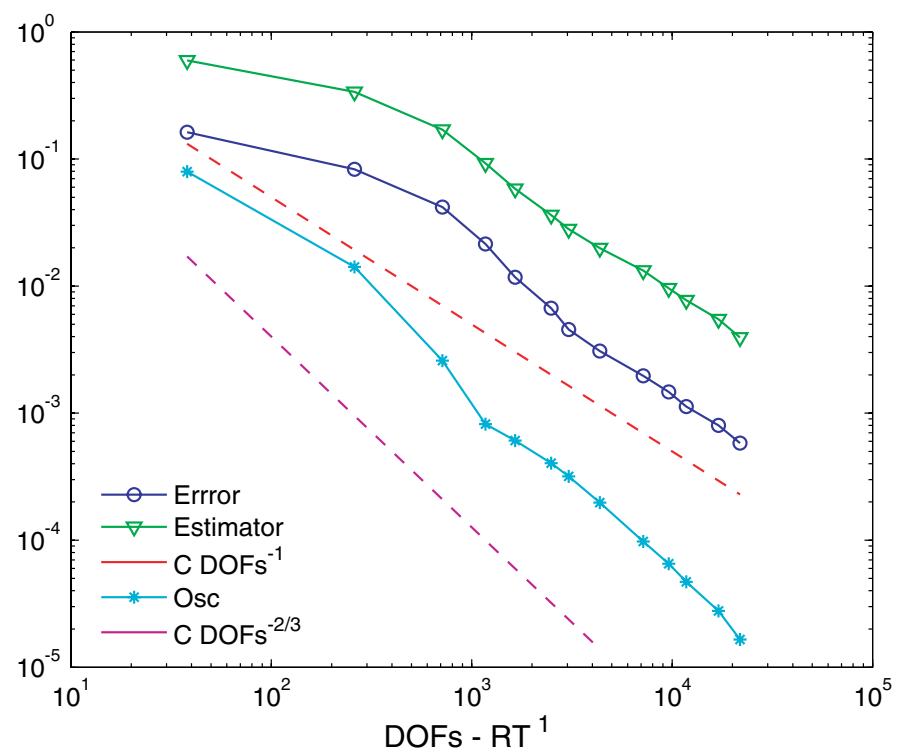

(b)

Fig. 4. L-shaped domain: Error, estimator $\eta_{k}$, and oscillation osc os $_{k}$ for (a) $\mathbb{R}^{0}$ and (b) $\mathbb{R} \mathbb{T}^{1}$. The optimal error decay is evident as well as the fact that oscillation is of higher order. 


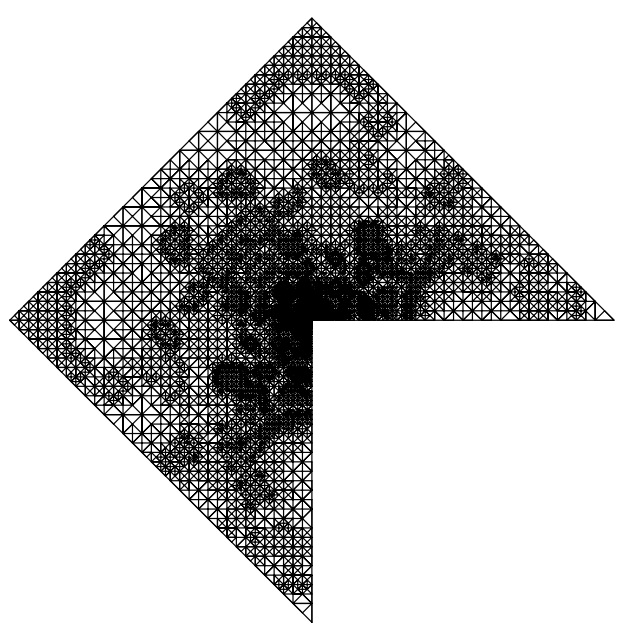

(a)

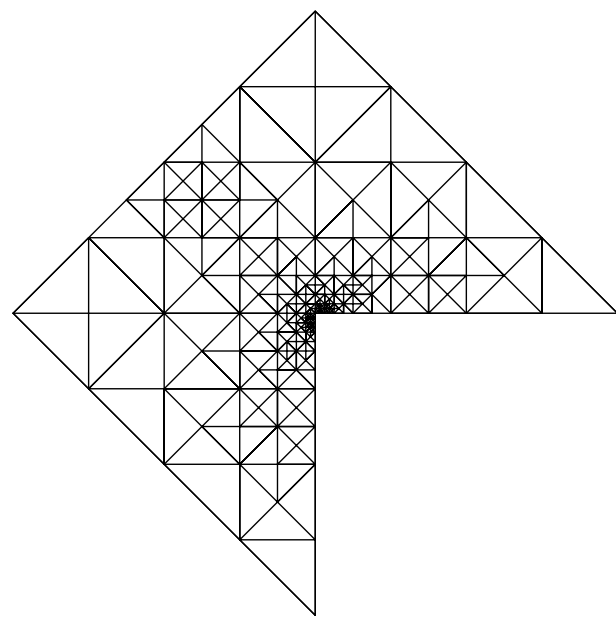

(b)

Fig. 5. L-shaped domain: Comparison of meshes generated by (a) $\mathbb{R} \mathbb{T}^{0}$ and (b) $\mathbb{R T}^{1}$ with approximately the same error $\left\|\mathbf{p}-\mathbf{P}_{k}\right\|_{\Omega} \approx 10^{-2}$.

as the number of DOFs grows; see Fig. 6(b). Iterations of PCG are rather stable in the quasi-monotone case, in agreement with theory. ${ }^{12}$ On the other hand, AFEM exhibits an optimal convergence rate, as documented in Fig. 6.

\subsection{Example 3: Crack domain}

Let $\Omega=\{|x|+|y|<1\} \backslash\{0 \leq x \leq 1, y=0\}$ be a domain with a crack, and let $\mathbf{p}$ be the solution of (1.1) with

$$
f_{1}=0, \quad \mathbf{f}_{\mathbf{2}}=\mathbf{1}, \quad \partial \Omega=\Gamma_{\mathrm{ess}} .
$$

We use AFEM with $\mathbb{R T}^{1}$ [Fig. 8(a)] and $\mathbb{B D M}^{1}$ [Fig. 8(b)], for which there are geometric singularities in each corner of $\Omega$. For $\mathbb{R}^{1}$ we perform experiments with oscillation of the same order as well as of higher order than the estimator, i.e. $L^{2}$ projection of $\mathbf{R}_{k \mid T}$ onto $\mathbb{R}^{0}(T)$, respectively $\mathbb{P}^{1}\left(T ; \mathbb{R}^{2}\right)$; see Remark 5.2. The adaptive method is insensitive to the order of oscillation because the estimator is similar in both cases for the same number of DOFs; see Fig. 8(a).

\section{Extension to Three Dimensions}

In this section, we extend convergence of AFEM to $3 \mathrm{~d}$. The main changes are related to the fact that the quasi-Helmholtz decomposition $\mathbf{q}=\boldsymbol{\Phi}+\operatorname{curl} \boldsymbol{\Psi}$ in $3 \mathrm{~d}$ hinges on a vector potential $\Psi \in H^{1}\left(\Omega ; \mathbb{R}^{3}\right)$. Since this influences the a posteriori analysis only slightly, the most important change is a $3 \mathrm{~d}$ version of Lemma 5.3 . We will only comment on the changes. 


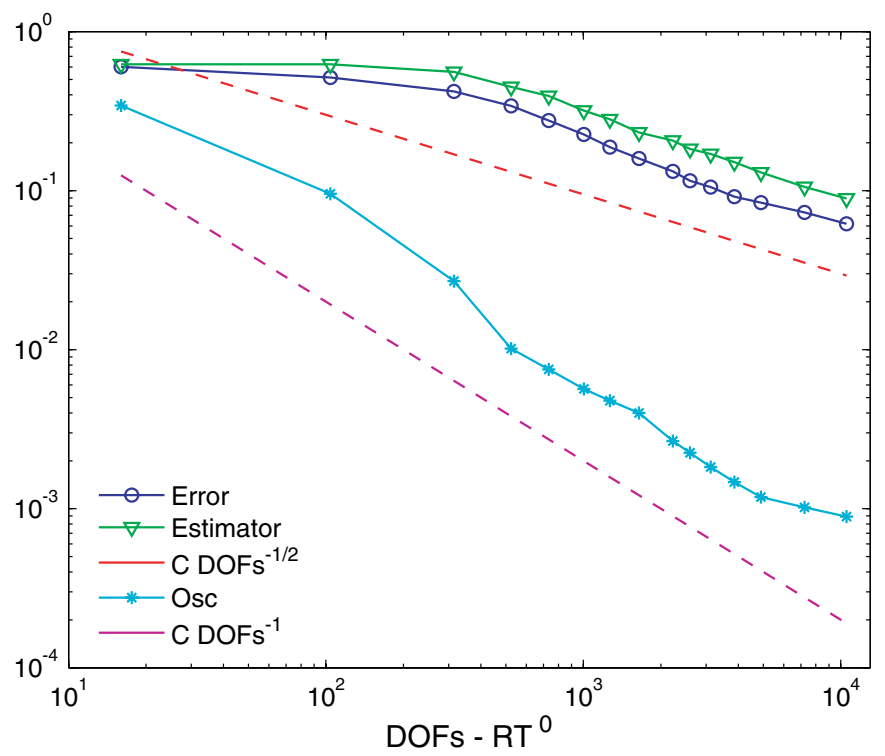

(a)

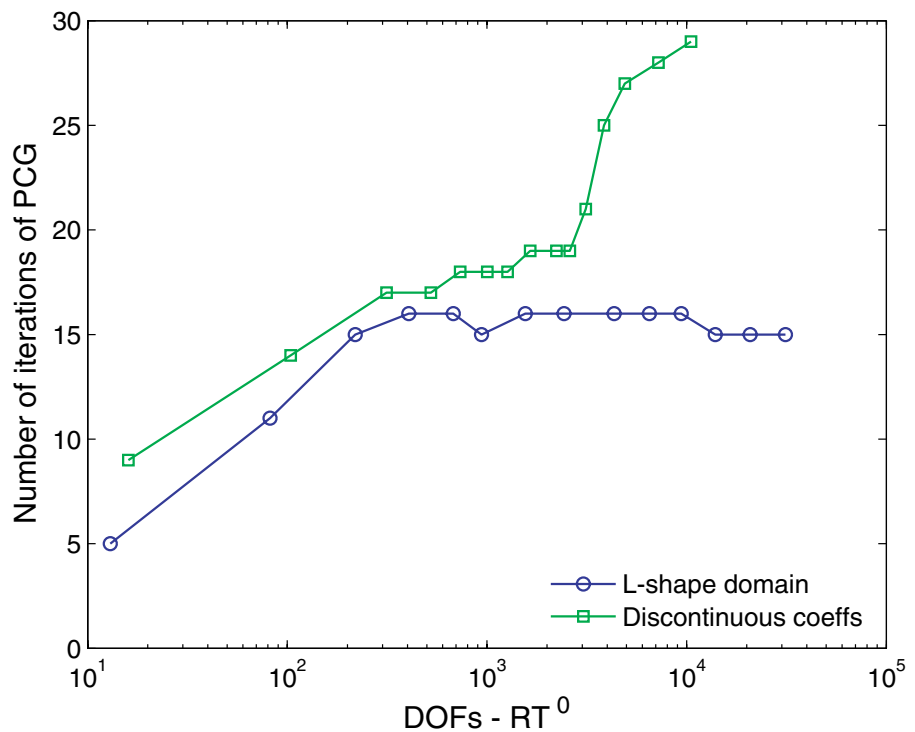

(b)

Fig. 6. (a) Discontinuous coefficients: Error estimator $\eta_{k}$, and oscillation osc ${ }_{k}$ for $\mathbb{R}^{0}$. (b) Number of iterations of PCG versus the number of DOFs. The number of iterations remains bounded as the coefficient matrix $A$ is the identity (Example 6.2: L-shape domain) but appears to grow linearly as the matrix $A$ exhibits a checkerboard pattern (Example 6.3: Discontinuous coefficients). 


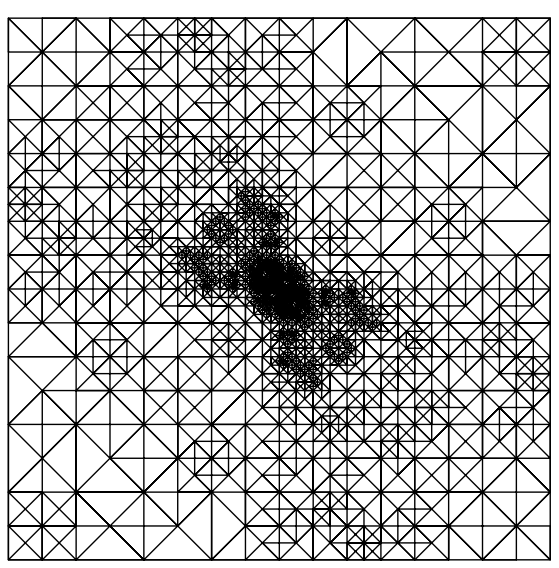

(a)

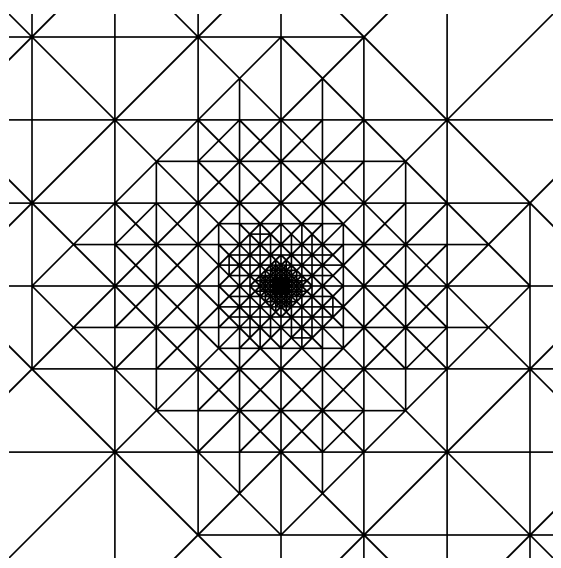

(c)

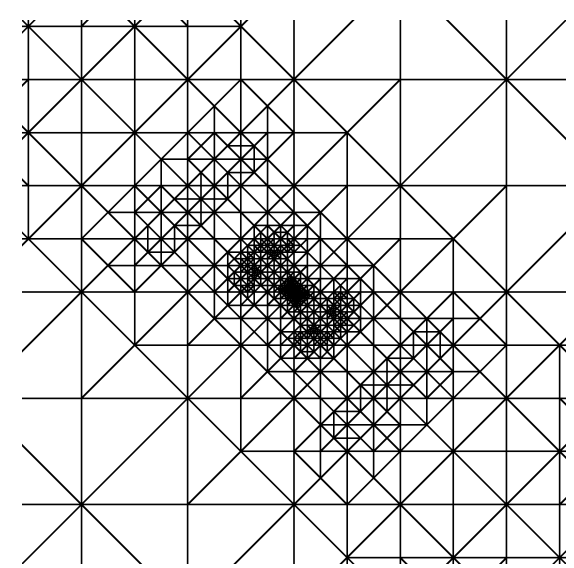

(b)

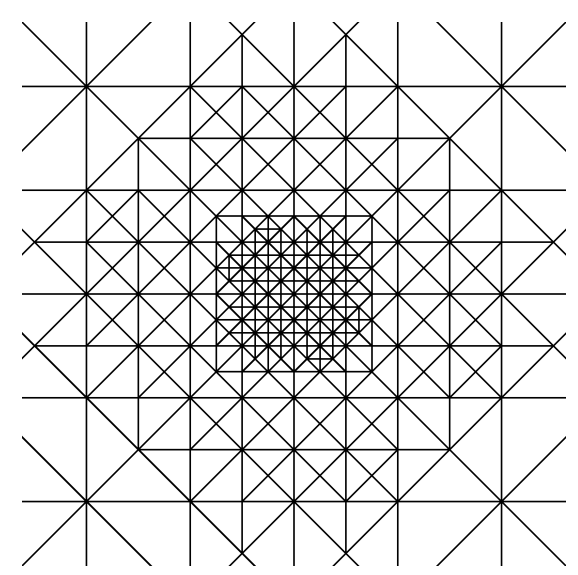

(d)

Fig. 7. Discontinuous coefficients: Adaptive grids for 14th iteration (10,524 DOFs): (a) full grid, (b) zooms to $\left(10^{-2}, 10^{-2}\right)^{2}$, (c) $\left(10^{-4}, 10^{-4}\right)^{2}$ and (d) $\left(10^{-5}, 10^{-5}\right)^{2}$.

\subsection{Approximation in $\mathbf{H}($ div $)$}

For the quasi-Helmholtz decomposition, define

$$
\operatorname{curl} \boldsymbol{\Psi}:=\nabla \times \boldsymbol{\Psi}:=\left[\partial_{x_{2}} \psi_{3}-\partial_{x_{3}} \psi_{2}, \partial_{x_{3}} \psi_{1}-\partial_{x_{1}} \psi_{3}, \partial_{x_{1}} \psi_{2}-\partial_{x_{2}} \psi_{1}\right]^{T} .
$$

The arguments of Lemma 5.1 are also true in $3 \mathrm{~d}$. However, in this case the boundary condition of the vector potential $\boldsymbol{\Psi}$, namely $\operatorname{curl} \boldsymbol{\Psi} \cdot \boldsymbol{\nu}_{\mid \Gamma_{\mathrm{ess}}}=0$, does not permit to choose $\Psi$ constant on $\Gamma_{\text {ess }}$. To circumvent this problem we now pose stronger assumptions on $\Omega$. Even though the following result can also be found in Ref. 14, we state here a more constructive proof suggested by Girault. 


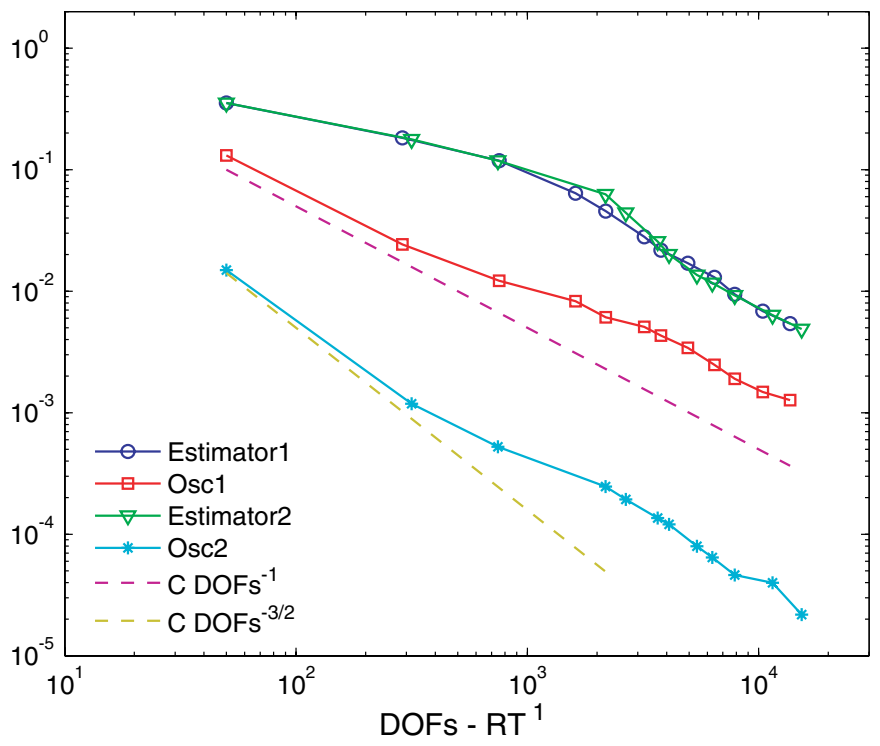

(a)

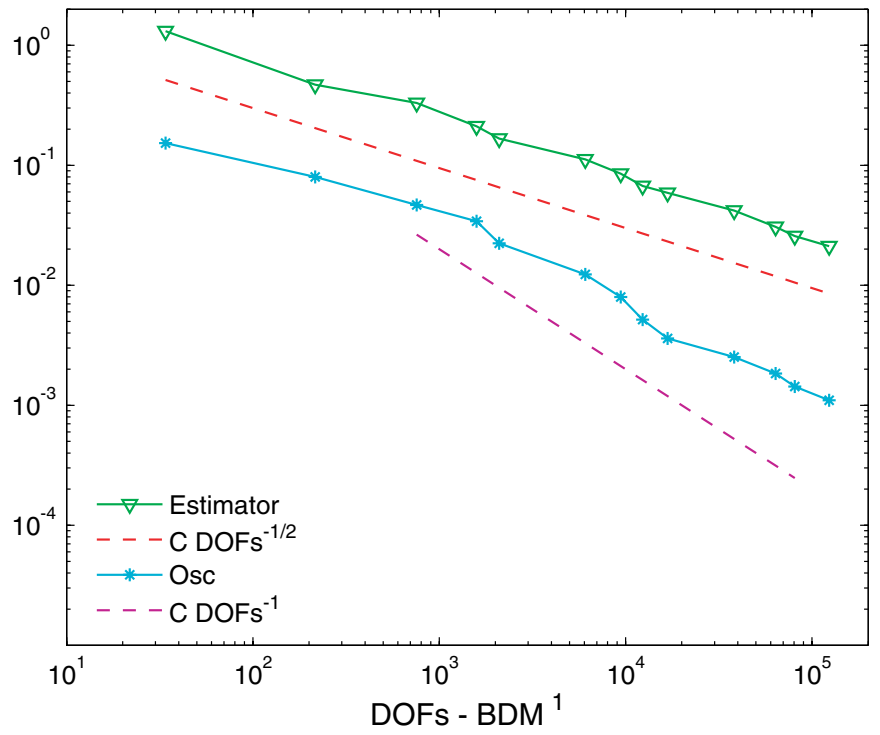

(b)

Fig. 8. Crack domain: Estimator $\eta_{k}$ and oscillation osc $c_{k}$ for (a) $\mathbb{R} \mathbb{T}^{1}$ and (b) $\mathbb{B D M} \mathbb{M}^{1}$. For $\mathbb{R}^{1}$, we have solved the problem using oscillation of same order as the estimator (Estimator1, Osc1) and oscillation of higher order (Estimator2, Osc2). Oscillation does not play a significant role in AFEM. 
Lemma 7.1. (Quasi-Helmholtz decomposition in $3 \mathrm{~d}$ ) Let $\Omega \subset \mathbb{R}^{3}$ be a bounded Lipschitz domain that is topologically equivalent to a ball and assume $\Gamma_{\text {nat }}=\emptyset$.

Then for all $\mathbf{q} \in \mathbb{Q}_{0}$ there exist $\boldsymbol{\Phi}, \boldsymbol{\Psi} \in H_{0}^{1}\left(\Omega ; \mathbb{R}^{3}\right)$, i.e. $\boldsymbol{\Phi}$ and $\boldsymbol{\Psi}$ have vanishing trace on $\partial \Omega$, such that

$$
\mathbf{q}=\boldsymbol{\Phi}+\operatorname{curl} \boldsymbol{\Psi} \quad \text { in } \Omega \quad \text { and } \quad\|\nabla \boldsymbol{\Phi}\|_{\Omega},\|\nabla \boldsymbol{\Psi}\|_{\Omega} \leq C\|\mathbf{q}\|_{\operatorname{div}, \Omega}
$$

Proof. We first observe that $q \in \mathbb{Q}_{0}$ implies $\langle\operatorname{div} \mathbf{q}, 1\rangle_{\Omega}=0$. Since $\Omega$ is connected, this implies the existence of a vector field $\boldsymbol{\Phi} \in H_{0}^{1}\left(\Omega ; \mathbb{R}^{3}\right)$ with

$$
\operatorname{div} \boldsymbol{\Phi}=\operatorname{div} \mathbf{q} \quad \text { in } \Omega \quad \text { and } \quad\|\boldsymbol{\Phi}\|_{\Omega},\|\nabla \boldsymbol{\Phi}\|_{\Omega} \preccurlyeq\|\mathbf{q}\|_{\operatorname{div}, \Omega} ;
$$

see Ref. 13 (Corollary I.2.4). We now consider a ball $B$ such that $\Omega$ is compactly contained in $B$. We define $\mathbf{v} \in H(\operatorname{div}, B)$ as

$$
\mathbf{v}:= \begin{cases}\mathbf{q}-\mathbf{\Phi} & \text { in } \Omega \\ 0 & \text { in } B \backslash \Omega .\end{cases}
$$

Then from Ref. 13 (Theorems I.3.4 and I.3.5) there exists $\hat{\boldsymbol{\Psi}} \in H^{1}\left(B ; \mathbb{R}^{3}\right)$ with $\hat{\boldsymbol{\Psi}} \cdot \boldsymbol{\nu}=0$ on $\partial B$ such that

$$
\mathbf{v}=\operatorname{curl} \hat{\Psi} \quad \text { and } \quad \operatorname{div} \hat{\Psi}=0
$$

Function $\hat{\boldsymbol{\Psi}}$ satisfies $\operatorname{curl} \hat{\boldsymbol{\Psi}}=0$ in $B \backslash \Omega$ and since $\Omega$ is topologically equivalent to a ball, $B \backslash \Omega$ is simply connected, and thus from Ref. 13 (Theorem I.2.9) there exists a function $\hat{s} \in H^{2}(B \backslash \Omega)$ such that $\hat{\mathbf{\Psi}}=\nabla \hat{s}$. Using Calderón's extension (Ref. 6, Theorem 12), Ref. $23 \hat{s}$ can be extended to the interior of $\Omega$, obtaining $s \in H^{2}(B)$ with

$$
\|\Delta s\|_{B} \preccurlyeq\|\nabla \hat{\Psi}\|_{D \backslash \Omega}
$$

We now define $\boldsymbol{\Psi}:=\hat{\boldsymbol{\Psi}}-\nabla s \in H_{0}^{1}\left(\Omega, \mathbb{R}^{3}\right)$, since $\boldsymbol{\Psi}=\mathbf{0}$ in $B \backslash \Omega$. Finally, from (7.3), (7.4), and definition of $\boldsymbol{\Psi}$ we obtain the quasi-Helmholtz decomposition (7.1),

$$
q=\Phi+\operatorname{curl} \hat{\Psi}=\Phi+\operatorname{curl} \Psi
$$

To conclude the proof we need to bound $\boldsymbol{\Psi}$ in terms of $\mathbf{q}$. Since $\boldsymbol{\Psi} \in H_{0}^{1}\left(\Omega ; \mathbb{R}^{3}\right)$, (Ref. 13, Theorem I.3.9) yields

$$
\|\nabla \boldsymbol{\Psi}\|_{\Omega}^{2} \leq\|\operatorname{curl} \boldsymbol{\Psi}\|_{\Omega}^{2}+\|\operatorname{div} \boldsymbol{\Psi}\|_{\Omega}^{2}=\|\boldsymbol{\Phi}-\mathbf{q}\|_{\Omega}^{2}+\|\Delta s\|_{\Omega}^{2}
$$

by (7.1) and $\operatorname{div} \boldsymbol{\Psi}=\operatorname{div}(\hat{\boldsymbol{\Psi}}-\nabla s)=-\Delta s$. By (7.2) the first term is bounded by $\|\boldsymbol{\Phi}-\mathbf{q}\|_{\Omega} \preccurlyeq\|\mathbf{q}\|_{\operatorname{div}, \Omega}$. For the second term we observe that $\hat{\boldsymbol{\Psi}} \cdot \boldsymbol{\nu}=0$ on $\partial B$ is 
hence, from Ref. 13 (Theorem I.3.9) and (7.5), we see that $\|\Delta s\|_{\Omega}^{2} \preccurlyeq\|\nabla \hat{\mathbf{\Psi}}\|_{B}^{2} \leq\|\operatorname{curl} \hat{\boldsymbol{\Psi}}\|_{B}^{2}+\|\operatorname{div} \hat{\Psi}\|_{B}^{2}=\|\operatorname{curl} \hat{\Psi}\|_{B}^{2}=\|\mathbf{\Phi}-\mathbf{q}\|_{\Omega}^{2} \preccurlyeq\|\mathbf{q}\|_{\operatorname{div}, \Omega}^{2}$ again invoking (7.2). The desired bound (7.1) for $\boldsymbol{\Psi}$ is thus proven.

Using the quasi-Helmholtz decomposition we construct an approximation $\mathbf{Q}_{k}$ of $\mathbf{q} \in \mathbb{Q}_{0}$ by

$$
\mathrm{Q}_{k}=\boldsymbol{\Phi}_{k}+\operatorname{curl} \boldsymbol{\Psi}_{k}:=\Pi_{k}^{n} \boldsymbol{\Phi}+\operatorname{curl} \mathcal{I}_{k} \boldsymbol{\Psi},
$$

where $\Pi_{k}^{n}$ denotes the natural interpolation operator defined for $\mathbb{R T}_{0}^{n}$, respectively $\mathbb{B D M}_{0}^{n}$, and $\mathcal{I}_{k}$ is the Scott-Zhang interpolation operator onto the vector-valued piecewise linear functions over $\mathcal{T}^{k}$ with vanishing trace, defined component-wise. By construction, $\operatorname{curl} \boldsymbol{\Psi}_{k}$ is piecewise constant and for any side $\sigma, \operatorname{curl} \boldsymbol{\Psi}_{k} \cdot \boldsymbol{\nu}$ only involves tangential derivatives of $\boldsymbol{\Psi}_{k}$. Hence, the normal component of $\operatorname{curl} \boldsymbol{\Psi}_{k}$ is continuous across interior sides and 0 for boundary sides which implies $\operatorname{curl} \boldsymbol{\Psi}_{k} \in \mathbb{R T}_{0}^{0}\left(\mathcal{T}^{k}\right) \subset \mathbb{Q}_{0}^{k}$. The approximation properties of $\Pi_{k}^{n}$ (Ref. 5, Proposition III.3.6) and $\mathcal{I}_{k}$ (Ref. 22, Theorem 4.1) allow us to prove the (local) interpolation estimates

$$
\begin{gathered}
\left\|h_{k}^{-1}\left(\boldsymbol{\Phi}-\boldsymbol{\Phi}_{k}\right)\right\|_{\Omega}+\left\|h_{k}^{-\frac{1}{2}}\left(\boldsymbol{\Phi}-\boldsymbol{\Phi}_{k}\right)\right\|_{\Sigma_{k}} \preccurlyeq\|\mathbf{q}\|_{\text {div }, \Omega}, \\
\left\|h_{k}^{-1}\left(\boldsymbol{\Psi}-\boldsymbol{\Psi}_{k}\right)\right\|_{\Omega}+\left\|h_{k}^{-\frac{1}{2}}\left(\mathbf{\Psi}-\boldsymbol{\Psi}_{k}\right)\right\|_{\Sigma_{k}} \preccurlyeq\|\mathbf{q}\|_{\operatorname{div}, \Omega} .
\end{gathered}
$$

\subsection{Error representation}

Recall the definition of the residuals $\mathbf{R}_{k}$ and $J_{k}$ in Sec. 5 and redefine $\mathbf{r}_{k}$ and $\mathbf{j}_{k}$ as follows:

$$
\begin{aligned}
& \mathbf{r}_{k \mid T}:=\operatorname{curl}\left(\mathbf{f}_{\mathbf{2}}-\mathbf{A P}_{\mathbf{k}}\right) \quad \mathbf{T} \in \mathcal{T}^{\mathbf{k}}, \\
& \mathbf{j}_{k \mid \sigma}:= \begin{cases}\llbracket\left(\mathbf{f}_{\mathbf{2}}-A \mathbf{P}_{\mathbf{k}}\right) \times \boldsymbol{\nu} \rrbracket_{\sigma} & \sigma \in \mathcal{S}_{\mathrm{int}}^{k}, \\
\mathbf{0} & \sigma \in \mathcal{S}_{\mathrm{ess}}^{k} .\end{cases}
\end{aligned}
$$

We proceed as with the $2 \mathrm{~d}$ calculations to derive the error representation

$$
a\left(\mathbf{p}-\mathbf{P}_{k}, \mathbf{q}\right)=\left\langle\mathbf{R}_{k}, \boldsymbol{\Phi}\right\rangle_{\Omega}+\left\langle J_{k}, \boldsymbol{\Phi} \cdot \boldsymbol{\nu}\right\rangle_{\Sigma_{k}}+\left\langle\mathbf{r}_{k}, \boldsymbol{\Psi}\right\rangle_{\Omega}+\left\langle\mathbf{j}_{k}, \boldsymbol{\Psi}\right\rangle_{\Sigma_{k}}
$$

for any $\mathbf{q}=\boldsymbol{\Phi}+\operatorname{curl} \boldsymbol{\Psi} \in \mathbb{Q}_{0}$ with $\boldsymbol{\Phi} \in \mathbb{Q}_{0}$ and $\boldsymbol{\Psi} \in H_{0}^{1}\left(\Omega ; \mathbb{R}^{3}\right)$ which is the starting point in the proof of the lower and upper bound.

\subsection{Upper bound}

We now define the error indicators as

$$
\eta_{k}^{2}(T):=\left\|h_{k} \mathbf{R}_{k}\right\|_{T}^{2}+\left\|h_{k}^{\frac{1}{2}}\left(J_{k}-\bar{J}_{k}\right)\right\|_{\partial T}^{2}+\left\|h_{k} \mathbf{r}_{k}\right\|_{T}^{2}+\left\|h_{k}^{\frac{1}{2}} \mathbf{j}_{k}\right\|_{\partial T}^{2},
$$

where $\bar{J}_{k \mid \sigma}$ is the $L_{2}(\sigma)$ projection of $J_{k \mid \sigma}$ onto $\mathbb{P}^{n}(\sigma)$ for $\sigma \in \mathcal{S}^{k}$. Then (7.8), the quasi-Helmholtz decomposition (7.1) in conjunction with Galerkin orthogonality and (7.7) establish the upper bound

$$
\left\|\mathbf{p}-\mathbf{P}_{k}\right\|_{\Omega}^{2} \leq C_{1} \eta_{k}^{2}\left(\mathcal{T}^{k}\right) .
$$


Introducing oscillation of $J_{k}-\bar{J}_{k}$ in the upper bound is possible by property (5.3a) of $\Pi_{k}^{n}$ in $3 \mathrm{~d}$; see Ref. 5 (Sec. III.3, p. 127).

\subsection{Local lower bound}

Similarly to the $2 \mathrm{~d}$ situation of Lemma 5.3 , the local lower bound for the lowest order discretization hinges on the construction of a suitable bubble function $\mathbf{b} \in \mathbb{B}(T)$ for given constant vector $\mathbf{q}$. Let $T$ be a tetrahedron that is refined three times. Then for any $\mathbf{q} \in \mathbb{R}^{3}$ there exists $\mathbf{b} \in \mathbb{B}(T)$, i.e. $\mathbb{R T}^{0}$ on the refinement of $T$ with zero normal trace, such that

$$
\|\mathbf{q}\|_{T}^{2} \preccurlyeq\langle\mathbf{q}, \mathbf{b}\rangle_{T} \quad \text { and } \quad\|\mathbf{b}\|_{T} \preccurlyeq\|\mathbf{q}\|_{T} .
$$

The proof mimics that of Lemma 5.3. For the invertible and affine map $F: \hat{T} \rightarrow T$, the scaled Jacobian is now $\mathfrak{A}:=|D F|^{-1 / 3} D F$. In $3 \mathrm{~d}$, the bisection of an element depends on its type $t \in\{0,1,2\} .{ }^{16,21}$ Therefore, we have to show invertibility of $\mathfrak{B}$ for the three corresponding refinements of the reference element $\hat{T}$. Let $\boldsymbol{\xi}_{i}=\mathbf{e}_{i}$, $i=1,2,3$, be the canonical basis of $\mathbb{P}^{0}\left(\hat{T} ; \mathbb{R}^{3}\right)$ and denote by $\boldsymbol{\Phi}_{1}, \boldsymbol{\Phi}_{2}, \boldsymbol{\Phi}_{3}$ the Raviart-Thomas basis functions associated with three interior faces of the refined $\hat{T}$; see Fig. 9. The matrices $\mathfrak{B}_{t}, t \in\{0,1,2\}$, are now given as

$$
\mathfrak{B}_{0}=\frac{1}{4}\left[\begin{array}{rrr}
1 & 0 & 0 \\
0 & 0 & 1 \\
0 & 1 & -1
\end{array}\right], \quad \mathfrak{B}_{1}=\frac{1}{4}\left[\begin{array}{lll}
1 & 0 & 0 \\
0 & 0 & 1 \\
0 & 1 & 0
\end{array}\right], \quad \mathfrak{B}_{2}=\frac{1}{8}\left[\begin{array}{rrr}
2 & 1 & 0 \\
0 & 1 & 2 \\
0 & -2 & 0
\end{array}\right],
$$

and the invertibility of $\mathfrak{B}_{t}$ follows from $\left|\mathfrak{B}_{t}\right|= \pm \frac{1}{64}, t=0,1,2$.

Moreover, the basis functions $\boldsymbol{\Phi}_{i}$ have to be chosen properly in 3d, compare with Remark 5.2. The most natural choice of $\boldsymbol{\Phi}_{i} \in \mathbb{B}(\hat{T}), i=1,2,3$, are the RaviartThomas basic functions depicted in Fig. 10, which yield a singular matrix $\mathfrak{B}$.

We use the same definition of oscillation terms except for $\mathbb{R T}_{0}^{1}$ where we let, for conciseness, $\overline{\mathbf{R}}_{k}$ be the piecewise $L_{2}$ projection onto $\mathbb{R} \mathbb{T}_{0}^{0}$ and use the bubble
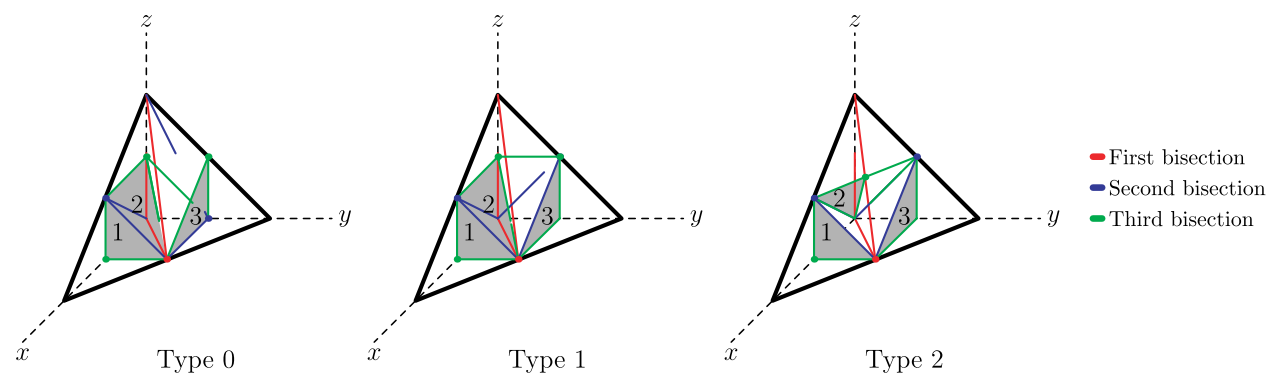

Fig. 9. Refinement of the reference tetrahedron of type 0,1 and 2 with three bisections, and nodes of Raviart-Thomas basis functions $\boldsymbol{\Phi}_{i}$ associated with three interior faces. The orientation of basis functions is such that $\boldsymbol{\Phi}_{i} \cdot[1,1,1]^{T}>0, i=1,2,3$. 

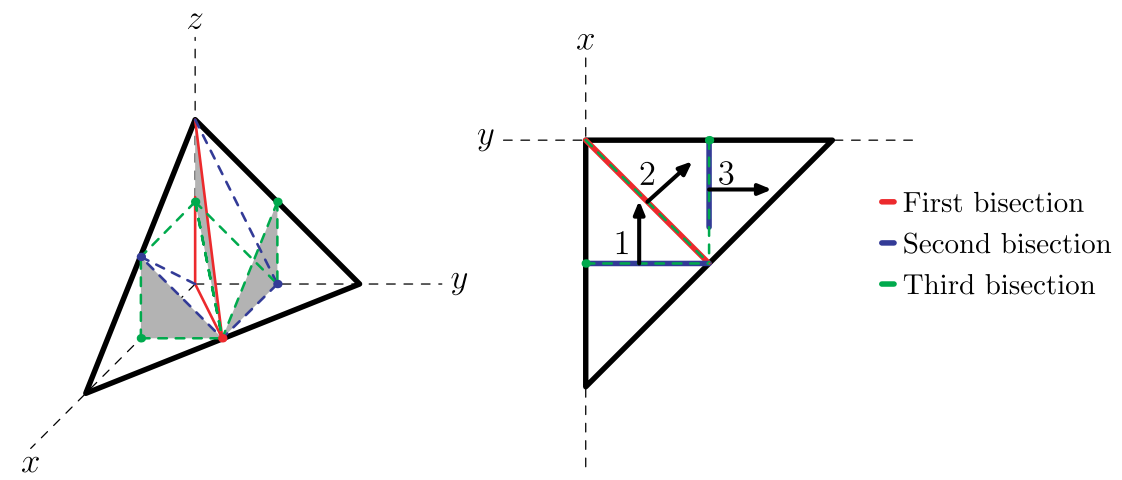

Fig. 10. Reference tetrahedron of type 0 refined with three bisections. Nodes and orientation of Raviart-Thomas basis functions associated with three interior sides which generate a singular matrix $\mathfrak{B}$. Therefore, these DOFs are inadequate to construct the bubble function $\mathbf{b} \in \mathbb{B}(\hat{T})$.

$\mathbf{b}:=\varphi_{T} \overline{\mathbf{R}}_{k}$ for $T \in \mathcal{T}^{k}$. With this modification the proof of the lower bound

$$
\bar{C}_{2} \eta_{k}^{2}(T) \leq\left\|\mathbf{P}_{k+1}-\mathbf{P}_{k}\right\|_{\omega_{T}}^{2}+\operatorname{osc}_{k}^{2}\left(\omega_{T}\right)
$$

is now the same as in $2 \mathrm{~d}$.

\subsection{Oscillation reduction}

The proof of oscillation reduction does not depend on the dimension. Consequently, Lemma 5.4 and Corollary 5.3 are also valid in $3 \mathrm{~d}$.

\subsection{Numerical experiment}

Let $\Omega$ be the L-shape domain shown in Fig. 11 and $\mathbf{p}:=A^{-1} u$ the solution of (1.1) with $u(\mathbf{x})=u(x, y, z)=\hat{u}(x, y)$,

$$
A=I, \quad f_{1}=1+u, \quad \mathbf{f}_{2}=\mathbf{0}, \quad g=u, \quad \partial \Omega=\Gamma_{\text {nat }},
$$

where $\hat{u}$ is the solution of Example 6.2. We use AFEM with $\mathbb{R T}^{0}$.

In this example, the discrete local lower bound for $T \in \mathcal{T}_{\text {est }}^{k}$ can be derived with just five bisections of $T$. No refinement of direct neighbors is needed. This is due to the following observations: Using the $\mathbb{R T}^{0}$ discretization, the element residual $\mathbf{R}_{k}$ is controled via a bubble function $\mathbf{b} \in \mathbb{B}(T)$ relying on three bisections of $T$. The bound for the second residual $\mathbf{r}_{k}$ would need an interior node inside $T$, but in this example $\mathbf{r}_{k}=\operatorname{curl}\left(\mathbf{f}_{\mathbf{2}}-\mathbf{P}_{\mathbf{k}}\right)=-\operatorname{curl} \mathbf{P}_{\mathbf{k}}=\mathbf{0}$; hence the interior nodes inside $T$ and its direct neighbors are not used. The estimate for $\mathbf{j}_{k}$ requires only nodes in the interior of all faces of $T$ which are created by bisecting $T$ five times. 


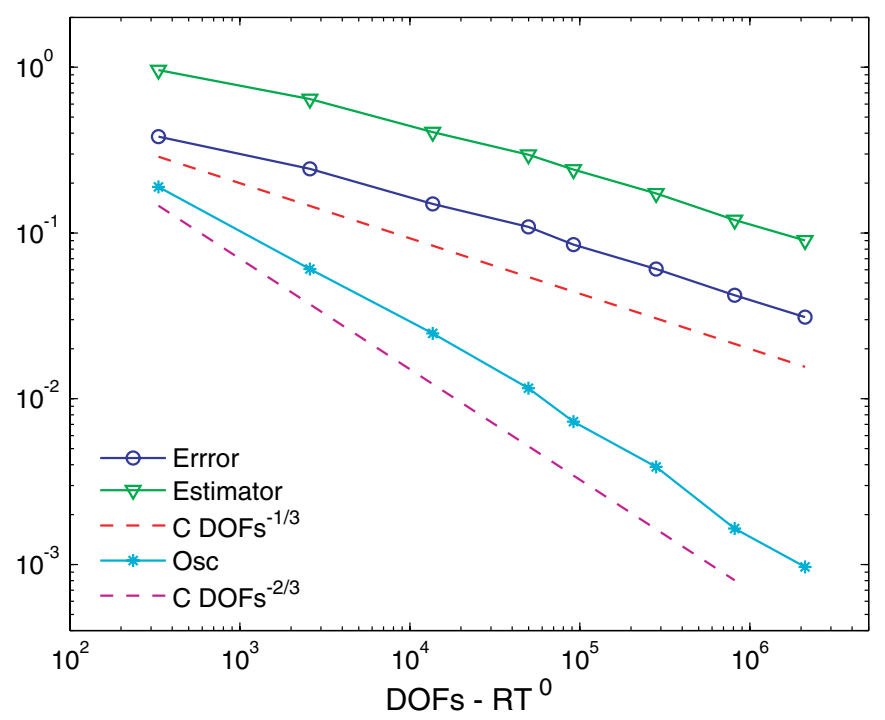

(a)

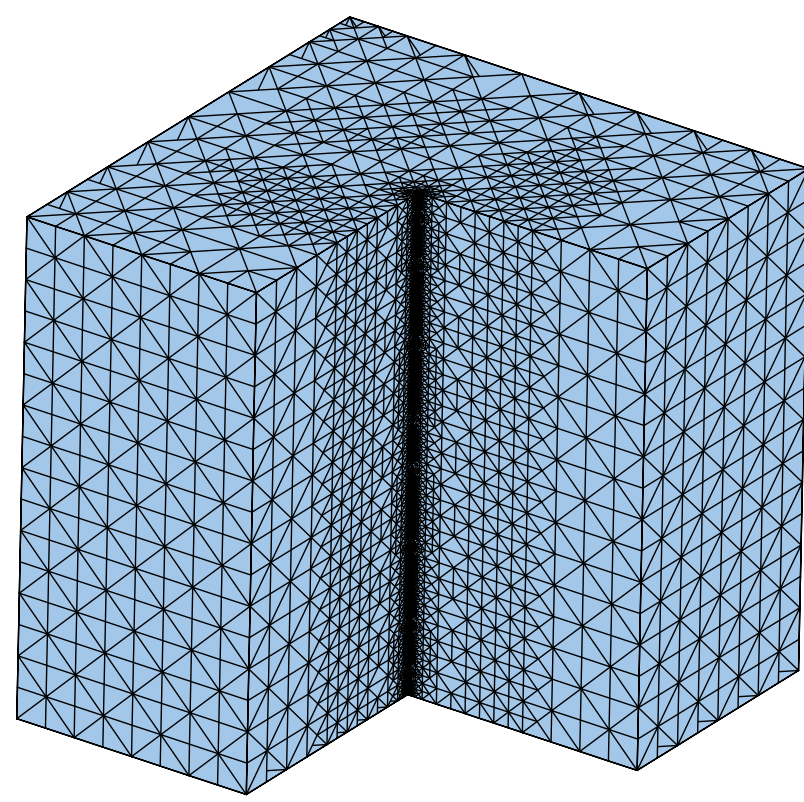

(b)

Fig. 11. L-shaped domain in 3d. (a) Error, estimator $\eta_{k}$ and oscillation osc ${ }_{k}$. (b) Mesh obtained in the sixth iteration, with 281,148 DOFs. 
MARK uses again $\theta_{\mathrm{osc}}=\theta_{\mathrm{est}}=0.4$, and REFINE applies five bisections for $T \in \mathcal{T}_{\text {est }}^{k}$ and one bisection for $T \in \mathcal{T}_{\text {osc }}^{k}$. The optimal error decay is evident from Fig. 11. We also observe from Fig. 11 that the oscillation is a higher order term with a minor role in the adaptive procedure.

\section{Acknowledgments}

We are thankful to V. Girault for suggesting the proof of Lemma 7.1. J.M.C. was partially supported by Grants CGL2004-06171-C03-03/CLI, Ministerio de Ciencia y Tecnología (Spain) and SA078A05, Junta de Castilla y León (Spain), FEDER funds (European Union), and NSF Grant DMS-0505454. R.H.N. was partially supported by NSF Grants DMS-0204670 and DMS-0505454. K.G.S. was partially supported by DAAD Grant "Efficient Finite Element Methods for Solid and Fluid Mechanics Computations".

\section{References}

1. A. Alonso, Error estimators for a mixed method, Numer. Math. 74 (1994) 385-395.

2. D. N. Arnold, R. S. Falk and R. Winther, Preconditioning in $H(d i v)$ and applications, Math. Comp. 66 (1997) 957-984.

3. E. Bänsch, Local mesh refinement in 2 and 3 dimensions, IMPACT Comput. Sci. Engrg. 3 (1991) 181-191.

4. E. Bänsch, P. Morin and R. H. Nochetto, An adaptive Uzawa FEM for the Stokes problem: Convergence without the inf-sup condition, SIAM J. Numer. Anal. 40 (2002) 1207-1229.

5. F. Brezzi and M. Fortin, Mixed and hybrid finite element methods in Springer Series in Computational Mathematics, Vol. 15 (Springer-Verlag, 1991).

6. A. P. Calderón, Lebesgue spaces of differentiable functions and distributions in Proc. Sympos. Pure Math., Vol. IV AMS (1961), pp. 33-49.

7. C. Carstensen, A posteriori error estimate for the mixed finite element method, Math. Comp. 66 (1997) 465-776.

8. J. M. Cascón, C. Kreuzer, R. H. Nochetto and K. G. Siebert, Quasi-optimal convergence rate for an adaptive finite element method, in preparation.

9. J. M. Cascón, R. H. Nochetto and K. G. Siebert, Convergence and optimality of AFEM in $\mathrm{H}(\mathrm{DIV})$, in preparation.

10. Z. Chen and J. Feng, An adaptive finite element method with reliable and efficient error control for linear parabolic problems, Math. Comp. 73 (2004) 1163-1197.

11. W. Dörfler, A convergent adaptive algorithm for Poisson's equation, SIAM J. Numer. Anal. 33 (1996) 1106-1124.

12. M. Dryja, M. V. Sarkis and O. B. Widlund, Multilevel Schwarz methods for elliptic problems with discontinuous coefficients in three dimensions, Numer. Math. 72 (1996) $313-348$.

13. V. Girault and P. A. Raviart, Finite Element Approximation of the Navier-Stokes Equations (Springer-Verlag, 1986).

14. R. Hiptmair and J. Xu, Nodal auxiliary space preconditioning in $H$ (curl) and $H$ (div) spaces, Research Report No. 2006-09, ETH Zürich.

15. R. B. Kellogg, On the Poisson equations with intersecting interfaces, Appl. Anal. 4 (1974/75) 101-129. 
16. I. Kossaczký, A recursive approach to local mesh refinement in two and three dimensions, J. Comput. Appl. Math. 55 (1994) 275-288.

17. K. Mekchay and R. H. Nochetto, Convergence of adaptive finite element methods for general second order linear elliptic PDE, SIAM J. Numer. Anal. 43 (2005) 1803-1827.

18. W. F. Mitchell, A comparison of adaptive refinement techniques for elliptic problems, ACM Trans. Math. Softw. 15 (1989) 326-347.

19. P. Morin, R. H. Nochetto and K. G. Siebert, Data oscillation and convergence of adaptive FEM, SIAM J. Numer. Anal. 38 (2000) 466-488.

20. P. Morin, R. H. Nochetto and K. G. Siebert, Convergence of adaptive finite element methods, SIAM Rev. 42 (2002) 631-658.

21. A. Schmidt and K. G. Siebert, Design of Adaptive Finite Element Software: The Finite Element Toolboox ALBERTA, LNCSE 42 (Springer, 2005).

22. L. R. Scott and S. Zhang, Finite element interpolation of nonsmooth functions satisfying boundary conditions, Math. Comp. 54 (1990) 483-493.

23. E. M. Stein, Singular Integrals and Differentiability Properties of Functions (Princeton Univ. Press, 1970).

24. B. Wohlmuth and R. H. W. Hoppe, A comparison of a posteriori error estimators for mixed finite element discretizations by Raviart-Thomas elements, Math. Comp. 68 (1999) 1347-1378. 